\title{
Actions of Discrete Amenable Groups and Groupoids on von Neumann Algebras
}

\author{
Dedicated to Professor M. Tomita on his 60th birthday
}

By

Colin E. SutherLAND* and Masamichi TAKeSAKI $\dagger^{* * *}$

\section{§0.1. Introduction}

The purpose of this paper is to provide a complete set of invariants, up to cocycle conjugacy, of the possible actions, $\alpha$, of a discrete amenable group $G$ on a semifinite injective von Neumann algebra $\mathscr{M}$. In the case where $\mathscr{M}=L^{\infty}(X, \mu)$, this amounts to giving invariants, up to conjugacy, for the non-singular actions of $G$ on a Lebesgue space $(X, \mu)$, a problem which is unsolved even for $G=\mathbb{Z}$; in the general case, the action of $G$ on the center of $\mathscr{M}$ itself appears as part of the invariant. If $\mathscr{M}$ is a factor and $G=\mathbb{Z}_{p}$ or $\mathbb{Z}$, the problem was solve by fundamental work of A. Connes in $[2,4]$; refining Connes' techniques, V. Jones, [9], resolved the case where $\mathscr{M}$ is a factor and $G$ is finite, and A. Ocneanu, [14], resolved the case where $\mathscr{M}$ is a factor and $G$ is amenable. Also, in [10], Jones and Takesaki gave a complete set of invariants for the case where $\mathscr{M}$ is no longer a factor, but $G$ is abelian. The main theorem of the present paper subsumes all these results, and depends crucially on the results of Ocneanu in [14], and the techniques developed by Jones and Takesaki in $[10]$ to handle the non-factor case.

For technical simplicity, we treat the case where the restriction of $\alpha$ to the center $\mathscr{Z}(\mathscr{M})$ is ergodic (i.e. $\alpha$ is centrally ergodic); we let

Communicated by H. Araki, August 7, 1984.

* Mathematics Department, University of New South Wales, P. O. Box 1. Kensington 2033, Australia.

** Mathematics Department, U. C. L. A., 405 Hilgard Avenue, Los Angeles, CA 90024, U. S. A.

$\dagger$ Research partially supported by NSF Grant MCS81-01589. 
$(g, x) \in G \times X \rightarrow g x \in X$ be a point realization, [12], of the restriction of $\alpha$ to $\mathscr{Z}(\mathscr{M})$. In the case where $G$ is abelian, the isotropy groups $G_{x}=\{g \in G: g x=x\}$ are essentially constant, and the main technical difficulty in handling the general case is that these isotropy groups can vary drastically with $x$. In particular, it has been necessary to adapt the "Cohomology Lemma" of [10] to a context where the coefficient groups for cocycles vary from point to point over $X$; this adaptation, while reasonably straight-forward, requires considerable preparation and has been treated in a separate paper, [16], written with the specific needs of the present work in mind. With Ocneanu's Theorem, [14], and this modified Cohomology Lemma at hand, our treatment parallels that of [10] for abelian groups quite closely.

As in [10], we actually work with actions of the groupoid $\mathscr{G}=$ $G \times X$ on a single factor $\mathscr{P}$ rather than with actions of $G$ on $\mathscr{M}$. It is relevant only that $\mathscr{G}$ is amenable in the sense of [21], and our main result is stated and proved in the context of actions of orbitally discrete measured ergodic groupoids, as in [6], on semifinite, injective factors; the corresponding result for actions of discrete amenable groups on semifinite injective algebras is then derived as a Corollary. In view of the fact that non-amenable groups can have amenable actions on Lebesgue spaces, this groupoid point of view actually provides a proper generalization of the case of actions of amenable groups.

The organization of the paper is as follows; in $\S 1$ we show precisely how the cocycle conjugacy problem for actions of $G$ may be interpreted as a cocycle conjugacy problem for actions of the associated groupoids $G \times X$. We then consider actions of a fixed ergodic, orbitally discrete groupoid $\mathscr{G}$ on a semifinite factor $\mathscr{P}$, define the invariants, and state the Main Theorem; our situation is somewhat more complicated than that of [10] in that we do not assume the existence of an invariant trace, and our invariant involves both the "characteristic invariant" of [10], and a "module" for the action as in [2]. The space of invariants is analyzed in $\S 2$, using a "semidirect product decomposition", $\mathscr{G}=\mathscr{H} \searrow_{s} \mathscr{K}$, of $\mathscr{G}$ as an "isotropy part" $\mathscr{H}$ and a "principal part", $\mathscr{K}$. Next, in $\S 3$, we show that all possible values of the invariants can occur for suitable actions of $\mathscr{G}$; in fact, to complete the proof, it is necessary to show that each of the invariants can be realized by an action which has an extra property. Techni- 
cally, this is the most complicated part of the paper, and uses the Cohomology Lemma in the construction. In $\S 4$ the proof of the Main Theorem is completed, using Ocneanu's Theorem and results on the stability at infinity of actions of discrete amenable groups, [14], and the Cohomology Lemma, [16], again. Finally in $\S 5$ we give some applications to the structure of von Neumann algebras associated to extensions of discrete amenable groups, and to the classification of coactions of discrete amenable groups on certain von Neumann algebras.

This work was conceived during the second author's visits to U.N.S.W. and A.N. U. in 1983, and gestated and delivered during the first author's visit to U.C. L. A. in 1984. Both authors are grateful to all Universities and Administrations involved in making the collaboration possible.

\section{§ 0.2. Notation and Terminology.}

Throughout, $G$ will denote a countable amenable group, $\mathscr{M}$ denotes a von Neumann algebra, and $\mathscr{P}$ a factor, both with separable preduals, [17]; $\mathscr{R}$ denotes the injective factor of type $\mathrm{II}_{1}$, and $\mathscr{R}_{0,1}$ denotes the injective factor of type $\mathrm{II}_{\infty}$. An action $\alpha$ of $G$ on $\mathscr{M}$ is a homomorphism $\alpha: g \in G \rightarrow \alpha_{g} \in \operatorname{Aut}(\mathscr{M})$, where $\operatorname{Aut}(\mathscr{M})$ is the group of $*_{\text {-auto- }}$ morphisms of $\mathscr{M}$, equipped with the usual topology and standard Borel structure, [8]. A cocycle for an action $\alpha$ of $G$ on $\mathscr{M}$ is a map $g \in G \rightarrow u_{g} \in \mathscr{U}(\mathscr{M})$ satisfying

$$
u_{g} \alpha_{g}\left(u_{h}\right)=u_{g h}, \quad g \in G, h \in G,
$$

where $\mathscr{U}(\mathscr{M})$ is the group of unitaries of $\mathscr{M}$; the space of such $\alpha$-cocycles is denoted $Z_{\alpha}^{1}(G ; \mathscr{U}(\mathscr{M}))$. Actions $\alpha$ and $\beta$ of $G$ on $\mathscr{M}$ and $\mathscr{N}$ are said to be cocycle conjugate if there is an isomorphism $\theta: \mathscr{M} \rightarrow \mathcal{N}$ with $\theta \cdot \alpha_{g} \cdot \theta^{-1}=\operatorname{Ad} u_{g} \bullet \beta_{g}$, where $u \in Z_{\beta}^{1}(G ; \mathscr{U}(\mathscr{N}))$; see $[10,14]$.

We denote by $\mathscr{G}$ a standard Borel groupoid, with unit space $X=\mathscr{G}^{(0)}$ and composable elements

$$
\mathscr{G}^{(2)}=\left\{\left(\gamma_{1}, \gamma_{2}\right) \in \mathscr{G} \times \mathscr{G}: s\left(\gamma_{1}\right)=r\left(\gamma_{2}\right)\right\},
$$

where $r$ and $s$ are the range and source maps respectively. Throughout, $(X, \nu)$ denotes a standard measure space. Frequently, we will need to invoke the existence of Borel maps on $X$ satisfing certain 
additional properties; typically, the existence of a suitable $\nu$-measurable map follows from a standard application of the von Neumann Measurable Selection Theorem, [17] and the existence of a suitable Borel map follows after deletion of a $\nu$-null set from $X$. For the most part, we will omit the arguments involved, although we make an exception for Lemma 4.3.

The reader is referred to [16] for details concerning Borel functors to Polish groups, and to [10] for further discussion and interpretation of the results.

\section{§1. Group Actions, and Ancillary Actions of Groupoids}

Let $\alpha$ be an action of $G$ on $\mathscr{M} ; \alpha$ is said to be centrally ergodic if the restriction of $\alpha$ to the centre $\mathscr{A}$ of $\mathscr{M}$ is ergodic. We consider only centrally ergodic actions. If $\mathscr{A}$ is atomic, $\alpha$ is induced from an action $\beta$ of some subgroup $H$ of $G$ on a factor $\mathscr{P}$, [19; Theorem 10.5] and the study of $\alpha$ is reduced to that of $\beta$. Henceforth, we assume $\mathscr{A}$ is non-atomic, that $\mathscr{M}$ is semifinite and injective, [3], and we let $\tau$ denote a faithful, normal, semifinite trace on $\mathscr{M}$. Let

$$
\{\mathscr{M}, \tau\}=\int_{X}^{\oplus}\left\{\mathscr{M}_{x}, \tau_{x}\right\} d \nu(x)
$$

be the central decomposition of $\{\mathscr{M}, \tau\},[17]$; since $\alpha$ is centrally ergodic, $\mathscr{M}$ is of type $\mathrm{I}_{n}, n=1,2, \ldots, \infty$, type $\mathrm{II}_{1}$ or type $\mathrm{II}_{\infty}$, so that $\mathscr{M} \cong \mathscr{A} \otimes \mathscr{P}$ with $\mathscr{P}$ a factor of type $\mathrm{I}_{n}, \mathscr{P}=\mathscr{R}$ or $\mathscr{P}=\mathscr{R}_{0,1}$, by [3]. As in [16], there is a map $(g, x) \in G \times X \rightarrow \alpha_{g, x} \in \operatorname{Aut}(\mathscr{P})$ determined by

$$
\left(\alpha_{g} T\right)(g x)=\alpha_{g, x}(T(x)) ;
$$

here $(g, x) \in G \times X \mapsto g x$ is a point realization of the restriction of $\alpha$ to $\mathscr{A}$, and $T=\int_{X}^{\oplus} T(x) d \nu(x) \in \mathscr{M}$. Note that $\mathscr{G}=G \times X$ becomes a standard Borel groupoid with $r(g, x)=g x, s(g, x)=x$ and $(g, h x)(h, x)=(g h, x)$; furthermore $\nu$ is quasi-invariant under $G$, so that $(\mathscr{G}, \nu)$ is, together with the Haar measure on $G$, a measured groupoid. Note that we may assume $(g, x) \rightarrow \alpha_{g, x}$ is Borel and satisfies

$$
\alpha_{g, h x} \cdot \alpha_{h, x}=\alpha_{g h, x} \quad \nu-a . e . \quad \text { for } g, h \in G .
$$

We thus have a Borel action of the measured groupoid $(\mathscr{G}, \nu)$ on $\mathscr{P}$, which will be referred to as the ancillary groupoid (and action) of the 
action $\alpha$.

In general, actions $\alpha$ and $\beta$ of an orbitally discrete measured groupoid $(\mathscr{G}, \nu),[6]$ on $\mathscr{P}$ are said to be cocycle-conjugate if there is a Borel map $\theta: x \in X \rightarrow \theta_{x} \in \operatorname{Aut}(\mathscr{P})$ and a Borel function: $r \in \mathscr{G} \rightarrow$ $u_{r} \in \mathscr{U}(\mathscr{P})$ with

i) $\theta_{r(\gamma)} \circ \alpha_{r} \cdot \theta_{s(\gamma)}^{-1}=\operatorname{Ad} u_{r} \cdot \beta_{r}$ a.e. on $\mathscr{G}$,

ii) $u_{\gamma} \beta_{\gamma}\left(u_{\gamma^{\prime}}\right)=u_{\gamma \gamma^{\prime}}$ a.e. on $\mathscr{G}^{(2)}$.

A function $u$ satisfying ii) above is called a $\beta$-cocycle, and the collection of all such (identified up to sets of measure zero) is denoted $Z_{\beta}^{1}(\mathscr{G}, \nu, \mathscr{U}(\mathscr{P}))$ or $Z_{\beta}^{1}(\mathscr{G}, \mathscr{U}(\mathscr{P}))$.

Proposition 1. 1. Let $\alpha, \beta$ be two actions of $G$ on $\mathscr{M}$ and $\mathcal{N}$ respectively, and let $\left(\mathscr{G}_{\alpha}, \nu_{\alpha}\right),\left(\mathscr{G}_{\beta}, \nu_{\beta}\right)$ be the ancillary groupoids with unit spaces $X$ and $Y$. Then $\alpha$ and $\beta$ are cocycle conjugate if and only if there is a Gequivariant isomorphism $\theta:\left(X, \nu_{\alpha}\right) \rightarrow\left(Y, \nu_{\beta}\right)$ such that the actions $(g, x) \rightarrow$ $\alpha_{g, x},(g, x) \rightarrow \beta_{g, \theta(x)}$ of $\mathscr{G}_{\alpha}$ are cocycle conjugate.

The proof is routine, and left to the reader; the same result holds with "conjugate" replacing "cocycle-conjugate" throughout. Also, note that if $\alpha$ and $\beta$ are cocycle conjugate, $\left(\mathscr{G}_{\alpha}, \nu_{\alpha}\right)$ is isomorphic to $\left(\mathscr{G}_{\beta}, \nu_{\beta}\right)$.

We now fix a (general) amenable, orbitally discrete, ergodic measured groupoid $(\mathscr{G}, \nu)$, and consider its actions on a semi-finite injective factor $\mathscr{P}$. Note that the "principal part" $\mathscr{K}$ of $\mathscr{G}$.

$$
\mathscr{K}=\{(x, y) \in X \times X: x=r(\gamma), y=s(\gamma) \text { for some } \gamma \in \mathscr{G}\},
$$

is a hyperfinite equivalence relation on $X$, by [21] and [5]; also if

$$
\mathscr{H}=\{\gamma \in \mathscr{G}: r(\gamma)=s(\gamma)\}
$$

is the "isotropy part" of $\mathscr{G}$, then the groups

$$
H_{x}=\{\gamma \in \mathscr{G}: r(\gamma)=s(\gamma)=x\}
$$

are (almost) all amenable by [21]. These two facts are crucial to the entire theory. A slight modification of Lemma 2.2.11 of [10] yields the existence of a Borel left inverse $k \in \mathscr{K} \rightarrow \gamma(k) \in \mathscr{G}$ to the surjection $\gamma \in \mathscr{G} \rightarrow(r(\gamma), s(\gamma))$, with $\gamma(k l)=\gamma(k) \gamma(l)$ on $\mathscr{K}^{(2)}$, which allows us to view $\mathscr{K}$ as a subgroupoid of $\mathscr{G}$. With $H_{x}$ as above, and

$$
H_{k}(h)=\gamma(k) h \gamma(k)^{-1} \quad \text { for } \quad h \in H_{s(k)},
$$

$H=\left(H_{x}, H_{k}\right)$ is a Borel functor from $\mathscr{K}$ to Polish groups as in $[16, \S 4]$.

If we set 


$$
\mathscr{H} * \mathscr{K}=\{(h, k) \in \mathscr{H} \times \mathscr{K}: s(h)=r(k)\},
$$

then $\mathscr{H} * \mathscr{K}$ becomes a standard Borel groupoid, written $\mathscr{H} \searrow_{s} \mathscr{K}$ with

$$
r(h, k)=r(k), s(h, k)=s(k), \text { and }(g, k)(h, l)=\left(g H_{k}(h), k l\right) .
$$

Evidently, $\mathscr{H} \rtimes_{s} \mathscr{K}$ is isomorphic as a (measured) groupoid with $(\mathscr{G}, \nu)$ under the map $(h, k) \in \mathscr{H} \rtimes_{s} \mathscr{K} \mapsto h k \in \mathscr{G}$, so that $\mathscr{G}$ is a "semidirect product" of $\mathscr{H}$ by an action of $\mathscr{K}$. In analyzing actions of $\mathscr{G}$ we will frequently restrict to each of $\mathscr{H}$ and $\mathscr{K}$, and analyze each piece separately- $\mathscr{H}$ via Ocneanu's Theorem, [14], and $\mathscr{K}$ via the Cohomology Lemma, [16].

We now turn to the invariants associated to an action: $\gamma \mapsto \alpha_{\gamma}$ of $(\mathscr{G}, \nu)$ on $\mathscr{P}$. Let

$$
\mathscr{N}_{\alpha}=\left\{n \in \mathscr{H}: \alpha_{n} \in \operatorname{Int}(\mathscr{P})\right\} .
$$

Note $\mathscr{N}_{\alpha}$ is a Borel subset of $\mathscr{H}$ and $\mathscr{N}_{x}=\mathscr{N}_{\alpha} \cap H_{x}$ is a normal Borel subgroup of $H_{x}$ for each $x$. Of course, if $\mathscr{P}$ is type I, $\mathscr{N}_{\alpha}=\mathscr{H}$. Choose a Borel function $n \in \mathscr{N}_{\alpha} \rightarrow u(n) \in \mathscr{U}(\mathscr{P})$ such that

$$
\alpha_{n}=\operatorname{Ad} u(n) \text { on } \mathscr{N}_{\alpha} ;
$$

as in [10], we have

$$
u(n) u(m)=\mu_{\alpha}(n, m) u(n m) \text { on } \mathscr{N}_{\alpha}^{(2)},
$$

and

$$
\alpha_{\gamma}\left(u\left(\gamma^{-1} n \gamma\right)\right)=\lambda_{\alpha}(n, \gamma) u(n) \text { on } \mathscr{G}^{(2)} \cap\left(\mathscr{N}_{\alpha} \times \mathscr{G}\right) \text { 。 }
$$

The pair $\left(\lambda_{\alpha}, \mu_{\alpha}\right)$ defines an "element" of $Z\left(\mathscr{G}, \mathscr{N}_{\alpha}, \mathbb{T}\right)$ as in [10], and a relative cohomology class $\chi_{\alpha}=\left[\lambda_{\alpha}, \mu_{\alpha}\right] \in \Lambda\left(\mathscr{G}, \mathscr{N}_{\alpha}, \mathbb{T}\right)$. (Note: The order of the variables is different from [10]). Let $\tau$ be a faithful normal semifinite trace on $\mathscr{P}$, normalized in the usual way if $\mathscr{P}$ is type $\mathrm{I}$ or type $\mathrm{II}_{1}$, and define $\Delta_{\alpha}(\gamma)$ by the relation

$$
\tau \cdot \alpha_{r}=\Delta_{\alpha}(\gamma) \tau \text {. }
$$

Note $\Delta_{\alpha}$ is a homomorphism from $\mathscr{G}$ to $\boldsymbol{R}_{+}^{*}$, which is trivial unless $\mathscr{P}$ is of type $\mathrm{II}_{\infty}$. We let $\delta_{\alpha}$ denote the cohomology class of $\Delta_{\alpha} \in Z^{1}\left(\mathscr{G}, \nu, \boldsymbol{R}_{+}^{*}\right)$ in $H^{1}\left(\mathscr{G}, \nu, \boldsymbol{R}_{+}^{*}\right)$ 。

We now can state our main result.

Theorem 1.2. Let $(\mathscr{G}, \nu)$ and $\mathscr{P}$ be as above. Then actions $\alpha$ and $\beta$ of $(\mathscr{G}, \nu)$ on $\mathscr{P}$ are cocycle conjugate if and only if $\mathscr{N}_{\alpha}=\mathscr{N}_{\beta}, \chi_{\alpha}=\chi_{\beta}$ and $\delta_{\alpha}=\delta_{\beta}$. 
The "only if" part follows from a direct calculation which is left to the reader. The converse will be accomplished in several parts.

\section{§2. The Structure of $\mathscr{A}(\mathscr{G}, \mathscr{N}, \mathbb{T})$}

In this section, we generalize Lemma 2.3.17 of [10].

Let $(\mathscr{G}, \nu)$ be an orbitally discrete, amenable measured groupoid, and let $\mathscr{G}=\mathscr{H} \searrow_{s} \mathscr{H}$ as described in $\S 1$. Note that if $\mathscr{N} \triangleleft \mathscr{H}$ is as in [10], so that $\mathscr{N}=\left\{N_{x}: x \in X\right\}$ with $N_{x}$ normal in $H_{x}, \dot{\cup} N_{x}$ Borel in $\dot{\cup} H_{x}$ and $H_{k}\left(N_{s(k)}\right)=N_{r(k)}$ for all $k \in \mathscr{K}$, then $\hat{N}=\left(\hat{N}_{x}, \hat{N}_{k}\right)$ is a Borel functor to compact abelian groups (by [16; Theorem 4.3]), where $\hat{N}_{x}$ is the Pontrjagin dual of $N_{x}$ and $\hat{N}_{k}$ is the transpose of the restriction of $H_{k}$ to $N_{s(k)} \subseteq H_{s(k)}$. We define a Borel functor $\hat{\mathscr{N}}^{\mathscr{H}}$ by

$$
\hat{\mathscr{N}}_{x}^{\mathscr{H}}=\left\{\varphi \in \hat{N}_{x}: \varphi\left(h^{-1} n h\right)=\varphi(n) \text { for all } h \in H_{x}, n \in N_{x}\right\} ;
$$

$\hat{\mathscr{N}}_{x}^{\mathscr{H}}$ is the restriction of $\mathscr{N}_{k}$ to $\hat{\mathscr{N}}_{x}^{\mathscr{H}}$. Let $\mathrm{Z}^{1}\left(\mathscr{K}, \hat{\mathscr{N}}^{\mathscr{H}}\right)$ be the group of $\hat{\mathscr{N}} \mathscr{H}$-cocycles (as in [16, Definition 5.4]); an element $\lambda$ of $Z^{1}(\mathscr{K}, \hat{\mathscr{N}} \mathscr{H})$ is a Borel function on $(\mathscr{N} \times \mathscr{K}) \cap \mathscr{G}^{(2)}$ satisfying

$$
\begin{aligned}
& \lambda\left(h n h^{-1}, k\right)=\lambda(n, k), \text { for }(n, h, k) \in \mathscr{G}^{(3)} \cap(\mathscr{N} \times \mathscr{H} \times \mathscr{K}), \\
& \lambda(n, k l)=\lambda(n, k)+\lambda\left(k^{-1} n k,\right) \text { for }(n, k, l) \in \mathscr{G}^{(3)} \cap(\mathscr{N} \times \mathscr{K} \times \mathscr{K}) .
\end{aligned}
$$

(In this section, abelian groups are written additively). If $\sigma$ is a Borel section of $\dot{U} \mathscr{\mathscr { N }}_{x}^{\mathscr{H}}$, we define

$$
\partial \sigma(n, k)=\langle n, \sigma\rangle-\left\langle k^{-1} n k, \sigma\right\rangle \text { on } \mathscr{G}^{(2)} \cap(\mathscr{N} \times \mathscr{K}),
$$

when $\langle$,$\rangle is the pairing of N_{x}$ and $\hat{N}_{x}$, and $\langle n, \sigma\rangle=\langle n, \sigma(r(n))\rangle$ Note $\partial \sigma \in Z^{1}(\mathscr{K}, \hat{\mathscr{N}} \mathscr{H})$; the group of all such elements is denoted $B^{1}(\mathscr{K}, \hat{\mathscr{N}} \mathscr{H})$, and the quotient by $H^{1}(\mathscr{K}, \hat{\mathscr{N}} \mathscr{H})$.

We now consider $Z(\mathscr{H}, \mathscr{N}, \mathbb{T})$ as defined in [10]. If $(\lambda, \mu) \in Z(\mathscr{H}, \mathcal{N}, \mathbb{T})$ and $k \in \mathscr{K}$, define $\left(k^{*} \lambda_{,} k^{*} \mu\right) \in Z\left(H_{r(k)}, N_{r(n)}, \mathbb{T}^{\prime}\right)$ by

$$
\begin{aligned}
& \left(k^{*} \lambda\right)(n, h)=\lambda\left(k^{-1} n k, k^{-1} h k\right), \quad(n, h) \in N_{r(k)} \times H_{r(k)}, \\
& \left(k^{*} \mu\right)(m, n)=\mu\left(k^{-1} m k, k^{-1} n k\right), \quad(m, n) \in N_{r(k)} \times N_{r(k)} .
\end{aligned}
$$

We say that $(\lambda, \mu) \in Z(\mathscr{H}, \mathcal{N}, \mathbb{T})$ is $\mathscr{K}$-invariant if

$$
\left(k^{*} \lambda, k^{*} \mu\right) \equiv\left(\lambda_{r(n)}, \mu_{r(k)}\right) \bmod B\left(H_{r(k)}, N_{r(k)}, \mathbb{T}^{\prime}\right)
$$

for all $k \in \mathscr{K} ; Z(\mathscr{H}, \mathscr{N}, \mathbb{T})^{\mathscr{H}}$ denotes the $\mathscr{K}$-invariant elements of $Z(\mathscr{H}, \mathscr{N}, \mathbb{T})$, and $\Lambda(\mathscr{H}, \mathscr{N}, \mathbb{T})^{\mathscr{H}}$ its image in $\Lambda(\mathscr{H}, \mathscr{N}, \mathbb{T})$.

We can now state the main result of this section. 
Theorem 2.1. In the above context, there is a natural short exact sequence:

$$
0 \rightarrow H^{1}(\mathscr{K}, \hat{\mathcal{N}} \mathscr{H}) \rightarrow \Lambda(\mathscr{G}, \mathcal{N}, \boldsymbol{T}) \rightarrow \Lambda(\mathscr{H}, \mathscr{N}, \boldsymbol{T})^{\mathscr{K}} \rightarrow 0 .
$$

Proof. Throughout this proof we will use $g, h$ for elements of $\mathscr{H}$, and $k, l$ for elements of $\mathscr{K}$ without further mention; equations are to be interpreted as asserting that both sides have a common domain and equality holds.

a) Injection of $H^{1}(\mathscr{K}, \hat{\mathscr{N}} \mathscr{H})$ : Let $\lambda \in Z^{1}(\mathscr{K}, \hat{\mathscr{N}} \mathscr{H})$ be given, and define $\tilde{\lambda}$ on $\mathscr{G}^{(2)} \cap(\mathscr{N} \times \mathscr{G})$ by

$$
\tilde{\lambda}(n, h k)=\lambda(n, k) \text {. }
$$

If $\tilde{\mu}(m, n)=0$ on $\mathscr{N}^{(2)}$, then using the identity

$$
\tilde{\lambda}(n, g k h l)=\tilde{\lambda}\left(n, g k h k^{-1} k l\right)=\lambda(n, k l)=\lambda(n, k)+\lambda\left(k^{-1} n k, l\right),
$$

one sees that $(\tilde{\lambda}, \tilde{\mu}) \in Z(\mathscr{G}, \mathcal{N}, \mathbb{T})$. If $\sigma: \mathcal{N} \rightarrow \boldsymbol{T}$ is a Borel function with $\sigma \mid X=1$ and $(\tilde{\lambda}, \tilde{\mu})=\left(\delta_{1} \sigma, \delta_{2} \sigma\right)$, then since $\delta_{2} \sigma=\tilde{\mu} \equiv 1, \sigma \in \hat{\mathcal{N}}$ (or, more precisely, is a Borel section of $\left.\dot{\cup} \hat{N}_{x}\right)$. Since $\tilde{\lambda}(n, h)=1$ and $\delta_{1} \sigma=\tilde{\lambda}$, $\sigma \in \hat{\mathscr{N}} \mathscr{H}$; thus $\lambda(n, k)=\sigma(n)-\sigma\left(k^{-1} n k\right)$, and $\lambda \in B^{1}(\mathscr{K}, \hat{\mathscr{N}} \mathscr{H})$, so that $H^{1}(\mathscr{K}, \hat{\mathscr{N}} \mathscr{H})$ is injected in $\Lambda(\mathscr{G}, \mathscr{N}, \boldsymbol{T})$.

b) Exactness at $\Lambda(\mathscr{G}, \mathcal{N}, \boldsymbol{T})$. Let $(\tilde{\lambda}, \tilde{\mu}) \in Z(\mathscr{G}, \mathcal{N}, \boldsymbol{T})$ and let $\lambda$ be the restriction of $\tilde{\lambda}$ to $\mathscr{H}$, and $\mu=\tilde{\mu}$. Clearly $(\lambda, \mu) \in Z(\mathscr{H}, \mathcal{N}, \boldsymbol{T})$. Also we have

$$
\begin{aligned}
\left(k^{*} \lambda\right)(n, h) & =\tilde{\lambda}\left(k^{-1} n k, k^{-1} h k\right) \\
& =\tilde{\lambda}\left(k^{-1} n k, k^{-1}\right)+\tilde{\lambda}(n, h k) \\
& =\lambda(n, h)+\tilde{\lambda}\left(h^{-1} n h, k\right)-\tilde{\lambda}(n, k) \\
& =\lambda(n, h)-\left(\delta_{1} \sigma_{k}\right)(n, h),
\end{aligned}
$$

where $\sigma_{k}(n)=\tilde{\lambda}(n, k)$. Similarly, we have

$$
\begin{aligned}
\left(k^{*} \mu\right)(m, n) & =\mu(m, n)+\tilde{\lambda}(m, k)+\tilde{\lambda}(n, k)-\tilde{\lambda}(m n, k) \\
& =\mu(m, n)-\left(\delta_{2} \sigma_{k}\right)(m, n),
\end{aligned}
$$

so that $(\lambda, \mu) \in Z(\mathscr{H}, \mathscr{N}, \boldsymbol{T})^{\mathscr{K}}$. Clearly, trivial elements of $Z(\mathscr{G}, \mathcal{N}, \boldsymbol{T})$ have trivial restrictions in $Z(\mathscr{H}, \mathscr{N}, \mathbb{T})$, as does the image of $H^{1}(\mathscr{K}, \hat{\mathscr{N}} \mathscr{H})$.

Conversely, suppose $(\tilde{\lambda}, \tilde{\mu}) \in Z(\mathscr{G}, \mathcal{N}, T)$ has a trivial restriction $(\lambda, \mu)$ in $Z(\mathscr{H}, \mathscr{N}, \boldsymbol{T})$. We may suppose $\lambda(n, h)=\mu(m, n)=0$. Since 


$$
\begin{aligned}
\lambda\left(h^{-1} n h, k\right) & =\lambda(n, h)+\lambda\left(h^{-1} n k, k\right)=\lambda\left(n, k k^{-1} h k\right) \\
& =\lambda(n, k)+\lambda\left(k^{-1} n k, k^{-1} h k\right)=\lambda(n, k),
\end{aligned}
$$

$\lambda \in Z^{1}(\mathscr{K}, \hat{\mathscr{N}} \mathscr{H})$ as required.

c) Exactness at $\Lambda(\mathscr{H}, \mathscr{N}, \mathbb{T})^{\mathscr{H}}$. Let $(\lambda, \mu) \in Z(\mathscr{H}, \mathscr{N}, \mathbb{T})^{\mathscr{K}}$, and choose $\sigma_{k}$ with $\left(k^{*} \lambda, k^{*} \mu\right)=(\lambda, \mu)-\left(\delta_{1} \sigma_{k}, \delta_{2} \sigma_{k}\right)$. We may suppose that $\sigma:(n, k)$ $\rightarrow \sigma(n, k)=\sigma_{k}(n)$ is Borel on $\mathscr{G}^{(2)} \cap(\mathscr{K} \times \mathscr{N})$, so that we have

$$
\begin{aligned}
& \lambda\left(k^{-1} n k, k^{-1} h k\right)=\lambda(n, h)-\sigma(n, k)+\sigma\left(h^{-1} n h, k\right), \\
& \mu\left(k^{-1} m k, k^{-1} n k\right)=\mu(m, n)-\sigma(m n, k)+\sigma(n, k)+\sigma(m, k) .
\end{aligned}
$$

Define $\tilde{\lambda}$ on $\mathscr{G}^{(2)} \cap(\mathcal{N} \times \mathscr{G})$ and $\tilde{\mu}$ by $\tilde{\mu}=\mu$ and

$$
\tilde{\lambda}(n, h k)=\lambda(n, h)+\sigma\left(h^{-1} n h, k\right) .
$$

Equations 2.3.5 and 2.3.8 of [10] are automatically satisfied by $(\tilde{\lambda}, \tilde{\mu})$, and equation 2.3 .6 routinely verified. As defined above, $\tilde{\lambda}$ does not necessarily satisfy equation 2.3 .7 of [10]- it is necessary to modify $\sigma$ to achieve this. To this end, note that

$$
\lambda-\delta_{1} \sigma_{k l}=(k l) * \lambda=k^{*}\left(l^{*} \lambda\right)=\lambda-\delta_{1} \sigma_{k}-k^{*}\left(\delta_{1} \sigma_{l}\right),
$$

from which we see

$$
\delta_{1} \sigma_{k l}=\delta_{1} \sigma_{k}+k^{*} \delta_{1} \sigma_{l} .
$$

Now, evaluating at $(n, h)$, we obtain

$$
\varphi\left(h^{-1} n h, k, l\right)=\varphi(n, k, l)
$$

where

$$
\varphi(n, k, l)=\sigma(n, k l)-\sigma(n, k)-\sigma\left(k^{-1} n k, l\right) .
$$

Similarly, since $\delta_{2} \sigma_{k l}=\delta_{2} \sigma_{k}+k^{*} \delta_{2} \sigma_{l}$, we obtain by evaluating at $(m, n) \in$ $\mathscr{N}^{(2)}$

$$
\varphi(m n, k, l)=\varphi(m, k, l)+\varphi(n, k, l) \text { 。 }
$$

Thus $\varphi(\cdot, k, l)$ takes values in $\hat{\mathscr{N}} \mathscr{H}$, and, from its form, we have $\varphi \in Z^{2}(\mathscr{K}, \hat{\mathscr{N}} \mathscr{H})$. Since $\mathscr{K}$ is hyperfinite, $\varphi$ is a coboundary i.e. there is a Borel function $\phi$ from $\mathscr{K}$ to $\hat{\mathscr{N}} \mathscr{H}$ with

$$
\varphi(n, k, l)=\psi(n, k)+\psi\left(k^{-1} n k, l\right)-\psi(n, k l)
$$

[Note: Since the coefficient groups $\hat{\mathscr{N}} \mathscr{H}$ vary from point to point along $X$, this does not follow from [7]; however, the usual proof of triviality of $Z^{2}(\mathscr{K}, A)$ using a single generator for $\mathscr{K}$ generalizes 
routinely to our context]. Define $\rho(n, k)$ by

$$
\rho(n, k)=\phi(n, k)+\sigma(n, k),
$$

so that

$$
\begin{aligned}
\rho(n, k l) & =\rho(n, k)+\rho\left(k^{-1} n k, l\right), \\
\lambda\left(k^{-1} n k, k^{-1} h k\right) & =\lambda(n, h)-\rho(n, k)+\rho\left(h^{-1} n h, k\right), \\
\mu\left(k^{-1} m k, k^{-1} n k\right) & =\mu(m, n)-\rho(m n, k)+\rho(m, k)+\rho(n, k) .
\end{aligned}
$$

Defining now

$$
\tilde{\lambda}(n, h k)=\lambda(n, h)+\rho\left(h^{-1} n h, k\right),
$$

we have

$$
\begin{aligned}
& \tilde{\lambda}(n, g k h l)-\tilde{\lambda}(n, g k)-\tilde{\lambda}\left(k^{-1} g^{-1} n g k, h l\right) \\
&= \lambda\left(n, g k h k^{-1}\right)+\rho\left(k h^{-1} k^{-1} g^{-1} n g k h k^{-1}, k l\right)-\lambda(n, g)-\rho\left(g^{-1} n g, k\right) \\
&-\lambda\left(k^{-1} g^{-1} n g k, h\right)-\rho\left(h^{-1} k^{-1} g^{-1} n g k h, l\right) .
\end{aligned}
$$

However, using 2.3.7 of [10], we have

$$
\lambda\left(n, g k h k^{-1}\right)=\lambda(n, g)+\lambda\left(g^{-1} n g, k h k^{-1}\right),
$$

and since

$$
\begin{aligned}
& \lambda\left(k^{-1} g^{-1} n g k, h\right)=\left(k^{*} \lambda\right)\left(g^{-1} n g, k h k^{-1}\right) \\
& =\lambda\left(g^{-1} n g, k h k^{-1}\right)-\rho\left(g^{-1} n g, k\right)+\rho\left(k h^{-1} k^{-1} g^{-1} n g k h k^{-1}, k\right),
\end{aligned}
$$

we obtain

$$
\begin{aligned}
& \tilde{\lambda}(n, g k h l)-\tilde{\lambda}(n, g k)-\tilde{\lambda}\left(k^{-1} g^{-1} n g k, h l\right) \\
&= \rho\left(k h^{-1} k^{-1} g^{-1} n g k h k^{-1}, k l\right)-\rho\left(g^{-1} n g, k\right)-\rho\left(h^{-1} k^{-1} g^{-1} n g k h, l\right) \\
&+\rho\left(g^{-1} n g, k\right)-\rho\left(k h^{-1} k^{-1} g^{-1} n g k h k^{-1}, k\right)=0,
\end{aligned}
$$

where we use the identity $\rho(m, k l)=\rho(m, k)+\rho\left(k^{-1} m k, l\right)$.

Since $\tilde{\lambda}$ restricts on $(\mathscr{N} \rtimes \mathscr{H}) \cap \mathscr{G}^{(2)}$ to $\lambda$, the proof is complete.

Remark 2.2. It is clear from the proof of exactness at $\Lambda(\mathscr{H}, \mathscr{N}, \mathbb{T})$ that if $\mathscr{G}=\mathscr{H} \rtimes_{s} \mathscr{K}$ is an arbitrary orbitally discrete measured groupoid with isotropy part $\mathscr{H}$ and principal part $\mathscr{K}$, no longer necessarily amenable, then there is an exact sequence

$$
0 \rightarrow H^{1}(\mathscr{K}, \hat{\mathscr{N}} \mathscr{H}) \rightarrow \Lambda(\mathscr{G}, \mathcal{N}, T) \rightarrow \Lambda(\mathscr{H}, \mathcal{N}, T)^{\mathscr{K}} \rightarrow H^{2}(\mathscr{K}, \hat{\mathscr{N}} \mathscr{H}) .
$$

In our situation, the hyperfiniteness of $\mathscr{K}$ forces $H^{2}(\mathscr{K}, \hat{\mathscr{N}} \mathscr{H})=0$.

Remark 2.3. Despite the splitting $\mathscr{G}=\mathscr{H} \rtimes_{s} \mathscr{K}$, the exact sequence of Theorem 2.1 does not necessarily split, in contrast to the situation 
in [10], where $\mathscr{G}=X \rtimes_{s} G$, with $G$ abelian.

\section{§. Construction of Model}

In this section, we show that any characteristic invariant $\chi=$ $[\lambda, \mu] \in \Lambda(\mathscr{G}, \mathcal{N}, \boldsymbol{T})$ and module $\delta: \mathscr{G} \mapsto \mathbb{R}_{+}^{*}$ appear as the invariant of some action $\alpha$ of $\mathscr{G}$ on a semi-finite injective factor $\mathscr{P}$. Depending upon $\chi$ and $\delta$, there is a natural restriction on the type of $\mathscr{P}$. For example, if $\delta$ is non-trivial, then only possible $\mathscr{P}$ is of type $\mathrm{II}_{\infty}$, and if $\mathscr{H} \neq \mathscr{N}$, then $\mathscr{P}$ can not be of type $\mathbb{I}$.

Theorem 3.1. Let $\mathscr{G}$ be an amenable orbitally discrete measured groupoid with $X=\mathscr{G}^{(0)}$ and associated ergodic principal groupoid $\mathscr{K}$, and let $\mathscr{H}$ be the isotropy subgroupaid of $\mathscr{G}$. Let $\mathscr{N}$ be a normal Borel subgroupoid of $\mathscr{G}$. If $\chi=[\lambda, \mu] \in \Lambda\left(\mathscr{G}, \mathscr{N}, \mathbb{T}^{\prime}\right)$ and $\delta$ is any Borel homomorphism from $\mathscr{G}$ to $\mathbb{R}_{+}^{*}$, such that $\delta(n)=1$ for every $n \in \mathscr{N}$, then there exists an action $m$ of $\mathscr{G}$ on a semi-finite injective factor $\mathscr{P}$ with the following properties:

a) The characteristic invariant $\chi_{m}$ and the module $\delta_{m}$ of $m$ are precisely the given $\chi$ and $\delta$;

b) For any $\varphi \in Z^{1}(\mathscr{K}, \widehat{\mathscr{H}})$ there exist a Borel map: $x \in X \mapsto \theta_{x} \in \operatorname{Aut}(\mathscr{P})$ and an m-cocycle $\{u(\gamma): \gamma \in \mathscr{G}\}$ such that (with $\gamma \in \mathscr{G}, g, h \in \mathscr{H}$, and $k, l \in \mathscr{K})$,

i) $\bmod \left(\theta_{x}\right)=1, x \in X$;

ii) $\theta_{r(\gamma)} \cdot m_{r} \cdot \theta_{s(\gamma)}^{-1}=\operatorname{Ad}(u(\gamma)) m_{\gamma}$.

iii) $u(g k) m_{g k}(u(h l))=\varphi \overline{(k, h)} u(g k h l)$.

Furthermore, if $\delta \equiv 1$, then $\mathscr{P}$ may be chosen to be an injective factor of type $I I_{1}$; otherwise $\mathscr{P}$ must be of type $I I_{\infty}$. If $\mathscr{N} \neq \mathscr{H}$, then $\mathscr{P}$ must be of type $I I$.

We shall construct $\{\mathscr{P}, m\}$ in several steps.

Observation 1 . We may assume $\delta \equiv 1$. In fact, if $\left\{\mathscr{P}_{1}, m^{\prime}\right\}$ realizes $\chi$ with $\delta_{m^{\prime}}=1$, then we set

$$
\mathscr{P}=\mathscr{P}_{1} \bar{\otimes} \mathscr{R}_{0,1}, \quad m_{r}=m_{r}^{\prime} \otimes n_{10 \mathrm{~g} \delta(\gamma)}, \quad \gamma \in \mathscr{G},
$$

where $\mathscr{R}_{0,1}$ is an injective factor of type $\mathbb{I}_{\infty}$ and $\left\{n_{t}\right\}$ is a one parameter automorphism group of $\mathscr{R}_{0,1}$ with $\bmod \left(n_{t}\right)=e^{t}$. If $m^{\prime}$ enjoys the property (b), then so does $m$. 
Observation 2. It is sufficient to consider the case that $\mathscr{P}=\mathscr{R}$ of type $\mathrm{II}_{1}$. If $\left\{\mathscr{R}, m^{\prime}\right\}$ realizes $\{\lambda, \mu, \delta \equiv 1\}$ with property (b), then $\left\{\mathscr{P}=\mathscr{R} \otimes \mathscr{L}(\mathscr{S}), m=m^{\prime} \otimes \iota\right\}$ realizes $\{\lambda, \mu, \delta \equiv 1\}$ with $\mathscr{P} \cong \mathscr{R}_{0,1}$.

Observation 3. Properties (a) and (b) can be handled separately. Suppose that $\left\{n_{r}\right\}$ and $\left\{p_{r}\right\}$ are actions of $\mathscr{G}$ on $\mathscr{R}$ such that

a) $\chi_{n}=\chi$ :

b) $\chi_{p}=1$ and $p$ has property (b).

Then we put

$$
m_{r}=n_{r} \bar{\otimes} p_{r}, \quad \gamma \in \mathscr{G}, \quad \text { on } \mathscr{R} \bar{\otimes} \mathscr{R} \cong \mathscr{R} .
$$

Clearly, $\{\mathscr{R}, m\}$ has the required properties.

Thus, we can approach the two problems (a) and (b) of Theorem 3. 1 separately.

Let $p$ be an action of $\mathscr{G}$ on $\mathscr{R}$ and let $\mathscr{C}(p)$ be the set of all those Borel functions $\varphi:(\mathscr{K} \times \mathscr{H}) \cap \mathscr{G}^{(2)} \mapsto \boldsymbol{T}$ for which there exist a Borel field $\left\{\theta_{x}\right\}=\left\{\theta_{x}^{\varphi}\right\}$ of automorphisms on $\mathscr{R}$ and a Borel function $u=u_{\varphi}$ : $\gamma \in \mathscr{G} \mapsto u_{\varphi}(\gamma) \in \mathscr{U}(\mathscr{R})$ satisfying the following conditions:

$$
\begin{aligned}
& \theta_{r(\gamma)} \cdot p_{\gamma} \cdot \theta_{s(\gamma)}^{-1}=\operatorname{Ad}(u(\gamma)) \cdot p_{r}, \gamma \in \mathscr{G} ; \\
& \left.u\right|_{\mathscr{H}} \in Z_{p}^{1}(\mathscr{H}) ;\left.\quad u\right|_{\mathscr{H}} \in Z_{p}^{1}(\mathscr{K}) ; \\
& u(g k) p_{g^{k}}(u(h l))=\frac{\varphi(k, h)}{\varphi(g k h l)}
\end{aligned}
$$

Proposition 3.2. $\mathscr{C}(p)$ is a group which is an invariant under cocycle conjugacy of $p$. Further, if $\varphi \in \mathscr{C}(p)$ and

$$
\varphi^{\prime}(k, h)=\chi_{r(k)}\left(k h k^{-1}\right) \varphi(k, h) \chi_{s(k)}(h)^{-1}
$$

for some Borel family of characters $\chi_{x} \in \hat{H}_{x}$, then $\varphi^{\prime} \in \mathscr{C}(p)$.

Before the proof, we state the following:

Remark 3.3. Any $\varphi \in \mathscr{C}(p)$ automatically enjoys the properties:

a) $\varphi(k, g h)=\varphi(k, g) \varphi(k, h)$;

b) $\varphi(k l, h)=\varphi(l, h) \varphi\left(k, l h l^{-1}\right)$.

Thus $\varphi \in Z^{1}(\mathscr{K}, \mathscr{H})$.

If $\left\{\varphi, \theta^{\varphi}, u_{\varphi}\right\}$ satisfies the above relations (1), (2) and (3), then we say that $\left\{\theta^{\varphi}, u_{\varphi}\right\}$ realizes $\varphi$.

Proof of Proposition 3.2. Suppose $\varphi \in \mathscr{C}(p)$ is realized by $\left\{\theta^{\varphi}, u_{\varphi}\right\}$ 
and that $q_{\gamma}=\sigma_{y} \bullet p_{r} \bullet \sigma_{x}^{-1}$ with $x=s(\gamma)$ and $y=r(\gamma)$. It then follows that $\left\{\sigma_{y} \circ \theta_{y}^{\varphi} \sigma_{y}^{-1}, \sigma_{y}\left(u_{\varphi}(\gamma)\right)\right\}$ realizes $\varphi$. Hence $\varphi \in \mathscr{C}(q)$. Suppose that $q_{r}=\operatorname{Ad}(v(\gamma)) \cdot p_{r}$ with $v \in Z_{p}^{1}(\mathscr{G})$. Then we have

$$
\begin{aligned}
\theta_{y} \cdot q_{\gamma} \cdot \theta_{x}^{-1} & =\operatorname{Ad}\left(\theta_{y}(v(\gamma)) \circ \theta_{y} \cdot p_{\gamma^{\circ}} \theta_{x}^{-1}\right. \\
& =\operatorname{Ad}\left(\theta_{y}(v(\gamma)) u(\gamma) v(\gamma)^{*}\right) q_{\gamma} .
\end{aligned}
$$

We claim that $\left\{\theta, \theta_{y}(v(\gamma)) u(\gamma) v(\gamma) *\right\}$ realizes $\varphi$ with respect to $q$. With $\gamma=g k$ and $\gamma^{\prime}=h l$ and $x=s(\gamma), y=r(\gamma)$, we compute:

$$
\begin{aligned}
& \theta_{y}(v(\gamma)) u(\gamma) v(\gamma) * q_{\gamma}\left(\theta_{x}\left(v\left(\gamma^{\prime}\right)\right) u\left(\gamma^{\prime}\right) v\left(\gamma^{\prime}\right) *\right) \\
& \quad=\theta_{y}(v(\gamma)) u(\gamma) p_{r}\left(\theta_{x}\left(v\left(\gamma^{\prime}\right)\right) u\left(\gamma^{\prime}\right) v\left(\gamma^{\prime}\right) *\right) v(\gamma)^{*} \\
& \quad=\theta_{y}(v(\gamma))\left(\theta_{y} \cdot p_{\gamma^{\circ}} \theta_{x}^{-1}\right)\left(\theta_{x}\left(v\left(\gamma^{\prime}\right)\right) u(\gamma) p_{\gamma}\left(u\left(\gamma^{\prime}\right)\right) v\left(\gamma \gamma^{\prime}\right)^{*}\right. \\
& \quad=\theta_{y}\left(v\left(\gamma \gamma^{\prime}\right)\right) \varphi(k, h) u\left(\gamma \gamma^{\prime}\right) v\left(\gamma \gamma^{\prime}\right) * .
\end{aligned}
$$

Hence with $w(\gamma)=\theta_{y}(v(\gamma)) u(\gamma) v(\gamma) *,\{\theta, w\}$ realizes $\varphi$ with respect to $q$. Thus $\mathscr{C}(p)$ is a cocycle conjugacy invariant as a set.

We now turn to the group structure. Suppose that $\varphi$ and $\psi$ are realized by $\{\theta, u\}$ and $\{\sigma, v\}$ relative to $p$ respectively. We then have, with $x=s(\gamma)$ and $y=r(\gamma), \gamma \in \mathscr{G}$,

$$
\begin{aligned}
\theta_{y}{ }^{\circ} \sigma_{y}^{-1} \cdot p_{r}{ }^{\circ} \sigma_{x} \cdot \theta_{x}^{-1} & =\theta_{y} \cdot \operatorname{Ad}\left(\sigma_{y}^{-1}(v(\gamma) *)\right) p_{r} \circ \theta_{x}^{-1} \\
& \left.=\operatorname{Ad}\left(\theta_{y} \sigma_{y}^{-1}\left(v(\gamma)^{*}\right)\right) u(\gamma)\right) p_{r} ;
\end{aligned}
$$

with $\gamma=g k$ and $\gamma^{\prime}=h l$, we calculate

$$
\begin{aligned}
& \left(\theta_{y}{ }^{\circ} \sigma_{y}^{-1}\right)\left(v(\gamma)^{*}\right) u(\gamma) p_{\gamma}\left(\left(\theta_{x} \circ \sigma_{x}^{-1}\right)\left(v\left(\gamma^{\prime}\right) *\right) u\left(\gamma^{\prime}\right)\right) \\
& =\theta_{y} \sigma_{y}^{-1}\left(v(\gamma)^{*}\right) \theta_{y} p_{\gamma} \theta_{x}^{-1}\left(\left(\theta_{x}{ }^{\circ} \sigma_{x}^{-1}\right)\left(v\left(\gamma^{\prime}\right) *\right)\right) u(\gamma) p_{\gamma}\left(u\left(\gamma^{\prime}\right)\right) \\
& =\theta_{y}\left(\sigma_{y}^{-1}(v(\gamma) *) p_{\gamma} \sigma_{x}^{-1}\left(v\left(\gamma^{\prime}\right) *\right)\right) \overline{\varphi(k, h)} u\left(\gamma \gamma^{\prime}\right) \\
& \left.=\theta_{y} \sigma_{y}^{-1}\left(v(\gamma) * \sigma_{y} p_{\gamma} \sigma_{x}^{-1}\left(v\left(\gamma^{\prime}\right) *\right)\right) \overline{\varphi(k, h}\right) u\left(r \gamma^{\prime}\right) \\
& =\theta_{y} \sigma_{y}^{-1}\left(p_{\gamma}\left(v\left(\gamma^{\prime}\right)^{*}\right) v(\gamma) * \overline{\varphi(k, h}\right) u\left(\gamma \gamma^{\prime}\right) \\
& =\theta_{y} \sigma_{y}^{-1}\left(\psi(k, h) v\left(r \gamma^{\prime}\right)^{*}\right) \varphi(k, h) u\left(\gamma \gamma^{\prime}\right) \\
& =\psi(k, h) \overline{\varphi(k, h)} \theta_{y}{ }^{\circ} \sigma_{y}^{-1}\left(v\left(\gamma \gamma^{\prime}\right) *\right) u\left(\gamma \gamma^{\prime}\right) .
\end{aligned}
$$

Thus $\bar{\phi} \varphi$ is realized by $\left\{\theta_{y} \sigma_{y}^{-1}, \theta \sigma^{-1}\left(v(\gamma)^{*}\right) u(\gamma)\right\}$.

Suppose $\varphi$ is realized by $\{\theta, u\}$ with respect to $p$, and $x \in X \rightarrow \chi_{x} \in \hat{H}_{x}$ is a Borel field of characters. Put

$$
u^{\prime}(h k)=\chi_{r(k)}(h) u(h k) .
$$

Clearly,

$$
\theta_{y} p_{r} \theta_{x}^{-1}=\operatorname{Ad}\left(u^{\prime}(\gamma)\right) \cdot p_{r}, \quad \gamma \in \mathscr{G},
$$

and $u^{\prime}$ satisfies (2). Now, we compute, with $\gamma=g k$ and $\gamma^{\prime}=h l$, 


$$
\begin{aligned}
& u^{\prime}(\gamma) p_{\gamma}\left(u^{\prime}\left(\gamma^{\prime}\right)\right)=\chi_{y}(g) u(\gamma) p_{\gamma}\left(\chi_{x}(h) u\left(\gamma^{\prime}\right)\right) \\
& =\chi_{y}(g) \chi_{x}(h) \overline{\varphi(k, h)} u\left(\gamma \gamma^{\prime}\right) \\
& =\chi_{y}(g) \chi_{x}(h) \overline{\varphi(k, h) \chi_{y}\left(g k h k^{-1}\right)} u^{\prime}\left(r \gamma^{\prime}\right) \\
& =\overline{\chi_{y}\left(k h k^{-1}\right) \varphi(k, h)} \chi_{x}(h) u^{\prime}\left(\gamma \gamma^{\prime}\right) \text {. }
\end{aligned}
$$

$\begin{array}{lll}\text { Thus } \varphi^{\prime} \text { defined by (4) belongs to } \mathscr{C}(p) \text {. } & \text { Q. E. D. }\end{array}$

We are now going to construct an action $p$ with $\mathscr{C}(p)=Z^{1}(\mathscr{K}, \widehat{\mathscr{H}})$. Define

$$
A(x)=H_{x} /\left[H_{x}, H_{x}\right],
$$

where $\left[H_{x}, H_{x}\right]$ means the commutator subgroup of $H_{x}$. Note that since $k H_{x} k^{-1}=H_{y}$ for $k \in \mathscr{K}$ with $x=s(k), y=r(k)$, there is, for each $k \in \mathscr{K}$, a natural isomorphism from $A(x)$ to $A(\gamma)$. Thus, we can identify $Z^{1}(\mathscr{K}, \hat{\mathscr{H}})$ with $Z^{1}(\mathscr{K}, \hat{A})$, where $\hat{A}$ is the Borel field: $x \in X \rightarrow A(x)^{\wedge}$ of compact abelian groups (see [16; Theorem 4.3]). We then have the following obvious lemma.

Lemma 3.4. Suppose $\pi: \mathscr{H} \rtimes_{s} \mathscr{K} \mapsto A \rtimes_{s} \mathscr{K}$ is the quotient map, and $q$ is an action of $A \rtimes_{s} \mathscr{K}$ such that $\mathscr{C}(q)=Z^{1}(\mathscr{K}, \hat{A})$. Then $p=q \cdot \pi$ is an action of $\mathscr{G}=\mathscr{H} \rtimes_{s} \mathscr{K}$ with $\mathscr{C}(p)=Z^{1}(\mathscr{K}, \widehat{\mathscr{H}})$.

Thus, it suffices to construct an action $q$ of $A \rtimes_{s} \mathscr{K}$ with $\mathscr{C}(p)=$ $Z^{1}(\mathscr{K}, \hat{A})$.

Choose a family $\left\{\Gamma_{x}\right\}$ of groups with the following properties:

Each $\Gamma_{x}$ is a dense countable subgroup of $\hat{A}(x)$;

$x \in X \mapsto \Gamma_{x}$ is a Borel field, i.e. $\dot{\cup} \Gamma_{x}$ is a Borel set in $\left.\dot{\cup} \hat{A}(x) .7\right)$

We note the existence of such a choice is guaranteed by $[16$, Theorem 4.3 and Theorem 1.2].

We can saturate $\left\{\Gamma_{x}\right\}$ with respect to the natural action of $\mathscr{K}$ on $\{\hat{A}(x)\}$, i. e.

$$
\langle\chi k, a\rangle=\langle\chi, k \cdot a\rangle,
$$

where $k \cdot a=k a k^{-1}$; thus we can assume:

The field $\left\{\Gamma_{x}\right\}=\Gamma$ is invariant under the action of $\mathscr{K}$.

By the Cohomology Lemma, [16; Theorem 5.5], every element of $Z^{1}(\mathscr{K}, \hat{A})$ is cohomologous to an element of $Z^{1}(\mathscr{K}, \Gamma)$. By Proposition 3.2 , it suffices to construct $q$ with $\mathscr{C}(q) \supset Z^{1}(\mathscr{K}, \Gamma)$. 
Define

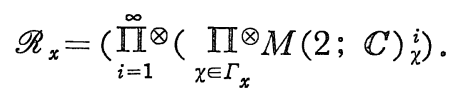

Let $\Gamma_{x}$ act on $\mathscr{R}_{x}$ via the shift:

$$
s_{\chi}^{x}\left(\prod_{i=1}^{\infty} \otimes\left(\prod_{\chi_{1} \in \Gamma_{x}}^{\otimes} a_{\chi_{1}}^{(i)}\right)\right)=\prod_{i=1}^{\infty} \otimes\left(\underset{\chi_{1} \in \Gamma_{x}}{\prod_{\chi \chi_{1}}} a^{(i)}\right)
$$

Also, if $t: \Gamma_{x} \mapsto \Gamma_{y}$ is an isomorphism, then there is an isomorphism $s_{t}: \mathscr{R}_{x} \mapsto \mathscr{R}_{y}$ via

$$
s_{t}\left(\prod_{i=1}^{\infty} \otimes\left(\prod_{\chi \in \Gamma_{x}}^{\otimes} a_{\chi}^{(i)}\right)\right)=\prod_{i=1}^{\infty} \otimes\left(\prod_{\chi \in \Gamma_{x}} \otimes a_{t(\chi)}^{(i)}\right) .
$$

The infinite tensor product guarantees the freeness of the action. It then follows that

$$
s_{t} \circ s_{\chi}^{x}=s_{t(\chi)}^{x} \bullet s_{t}, \chi \in \Gamma_{x} .
$$

Thus, we have a family of covariant systems $\left\{\mathscr{R}_{x}, s^{x}, \Gamma_{x}\right\}$, coherent with respect to $\mathscr{K}$. Put

$$
\mathscr{P}_{x}=\mathscr{R}_{x} \rtimes_{s} \Gamma_{x}, \quad x \in X,
$$

and let $p$ be the dual action of $B_{x}=\hat{\Gamma}_{x}$ on $\mathscr{P}_{x^{\circ}}$ Since $s^{x}$ is free, $\mathscr{P}_{x}$ is an injective factor of type II. Note that we have a canonical inclusion: $A(x) \longleftrightarrow B_{x}$, which is faithful and with dense range. For each $\chi \in \Gamma_{x}$, let $\{u(x, \chi)\}$ be the unitary representation of $\Gamma_{x}$ in $\mathscr{P}_{x}$ associated with the crossed product (13).

Lemma 3.5. For each $k \in \mathscr{K}, k: x \rightarrow y$, the map

$$
p_{k}:\left\{\begin{array}{l}
a \in \mathscr{R}_{x} \mapsto s_{k}(a) \in \mathscr{R}_{y}, \\
u(x, \chi) \in \mathscr{P}_{x} \mapsto u\left(y, \chi k^{-1}\right) \in \mathscr{P}_{y},
\end{array}\right.
$$

extends uniquely to an isomorphism, denoted again by $p_{k}$ of $\mathscr{P}_{x}$ onto $\mathscr{P}_{y}$ which satisfies

$$
p_{k} \circ p_{a}=p_{k a k} 1^{\circ} p_{k}, \quad a \in A(x) .
$$

The proof is just routine, so we leave it to the reader. The identity (15) gives us an action $p$ of $\mathrm{A} \rtimes_{s} \mathscr{K}$ on $\mathscr{P}=\left\{\mathscr{P}_{x}\right\}$ by

$$
p_{(a, k)}=p_{a} \cdot p_{k}, \quad(a, k) \in A \rtimes_{s} \mathscr{K} .
$$

We now set up the following system: 


$$
\begin{aligned}
& \mathscr{Q}_{x}=\prod_{n=-\infty}^{\infty} \otimes \mathscr{P}_{x, n}, \\
& q_{a}=\prod_{n=-\infty}^{\infty} p_{a}^{n}, \quad q_{k}=\prod_{n=-\infty}^{\infty} p_{k}^{n}, \\
& \theta_{x}=\text { the shift to the left on } \mathscr{Q}_{x}, \\
& \beta_{\chi}=\prod_{n \leqq 0}^{\otimes} 1_{n}^{\otimes}\left(\prod_{n=1}^{\infty} \mathrm{Ad} u_{n}(x, \chi)\right), \\
& v(\chi)=\prod_{n \leqq-1}^{\otimes} 1_{n} \otimes u_{0}(x, \chi) \otimes \Pi_{n \geqq 1}^{\otimes} 1_{n},
\end{aligned}
$$

where index $n$ means to repeat the same thing. Then we have the following formulae:

$$
\begin{aligned}
& q_{k} \cdot q_{a}=q_{k \cdot a} \cdot q_{k}, \\
& q_{a} \cdot \beta_{\chi}=\beta_{\chi} \cdot q_{a}, \\
& \beta_{\chi} \cdot q_{k}=q_{k} \cdot \beta_{\chi \cdot k}, \\
& \theta_{x} \cdot q_{a} \cdot \theta_{x}^{-1}=q_{a}, \\
& \theta_{x} \cdot \beta_{\chi} \cdot \theta_{x}^{-1}=\operatorname{Ad}\left(v_{\chi}\right) \cdot \beta_{\chi}, \\
& q_{a}\left(v_{\chi}\right)=\langle a, \chi\rangle n_{\chi}, \\
& q_{k}\left(v_{\chi}\right)=v_{\chi \cdot k}-1 \cdot
\end{aligned}
$$

For a given $\varphi \in Z^{1}(\mathscr{K}, \Gamma)$, we define an action $q^{\varphi}$ of $A \rtimes_{s} \mathscr{K}$ on $\mathscr{Q}=\left\{\mathscr{Q}_{x}\right\}$ by

$$
q_{(a, k)}^{\varphi}=q_{a} \bullet q_{k} \cdot \beta_{\varphi(k)}, \quad(a, k) \in A \rtimes_{s} \mathscr{K} .
$$

Lemma 3.6. $\varphi \in \mathscr{C}\left(q^{\varphi}\right)$.

Proof. With $k \in \mathscr{K}, k: x \rightarrow y$, we compute

$$
\begin{aligned}
\theta_{y} \cdot q_{(a, k)}^{\varphi} \cdot \theta_{x}^{-1} & =\theta_{y} \cdot q_{a} \cdot q_{k} \cdot \theta_{x}^{-1} \cdot \theta_{x} \cdot \beta_{\varphi(k)} \cdot \theta_{x}^{-1} \\
& =q_{a} \bullet q_{k} \cdot \operatorname{Ad}(v(\varphi(k))) \cdot \beta_{\varphi(k)} \\
& =\operatorname{Ad}\left(q_{a} \bullet q_{k}(v(\varphi(k)))\right) q_{a} \bullet q_{k} \cdot \beta_{\varphi(k)} \\
& =\operatorname{Ad} w(a, k) \cdot q_{(a, k)}^{\varphi},
\end{aligned}
$$

where $w(a, k)=q_{a} \cdot q_{k}(v(\varphi(k)))$. We then have

$$
\begin{aligned}
& w(a, k) q_{(a, k)}^{\varphi}\left(w\left(a^{\prime}, k^{\prime}\right)\right) \\
& \quad=q_{a} \bullet q_{k}(v(\varphi(k))) q_{a} \cdot q_{k} \cdot \beta_{\varphi(k)}\left(q_{a}{ }^{\prime} \cdot q_{k}{ }^{\prime}\left(v\left(\varphi\left(k^{\prime}\right)\right)\right)\right) \\
& \quad=q_{a} \cdot q_{k}\left(v(\varphi(k)) \beta_{\varphi(k)}\left(q_{a^{\prime}} \cdot q_{k^{\prime}}\left(v\left(\varphi\left(k^{\prime}\right)\right)\right)\right) .\right.
\end{aligned}
$$

But we have

$$
\begin{aligned}
w\left(a k \cdot a^{\prime}, k k^{\prime}\right) & =q_{a} \cdot q_{k \cdot a^{\prime}} \cdot q_{k k^{\prime}}\left(v\left(\varphi\left(k k^{\prime}\right)\right)\right) \\
& =q_{a} \cdot q_{k} \cdot q_{a^{\prime}} \cdot q_{k^{\prime}}\left(v\left(\left(\varphi(k) \cdot k^{\prime}\right) \varphi\left(k^{\prime}\right)\right)\right)
\end{aligned}
$$




$$
\begin{aligned}
& =q_{a} \circ q_{k}\left[q_{a^{\prime}} q_{k^{\prime}}\left(v\left(\varphi(k) \circ k^{\prime}\right)\right) q_{a^{\prime}} \circ q_{k^{\prime}}\left(v\left(\varphi\left(k^{\prime}\right)\right)\right)\right] \\
& =q_{a^{\circ}} q_{k^{\circ}} q_{a^{\prime}}(v(\varphi(k))) q_{a} \circ q_{k^{\circ}} q_{a^{\prime}} \circ q_{k^{\prime}}\left(v\left(\varphi\left(k^{\prime}\right)\right)\right) \\
& =\left\langle a^{\prime}, \varphi(k)\right\rangle w(a, k) q_{a} \circ q_{k} \circ \beta_{\varphi(k)} \circ q_{a^{\prime}} \circ q_{k^{\prime}}\left(v\left(\varphi\left(k^{\prime}\right)\right)\right) \\
& =\left\langle a^{\prime}, \varphi(k)\right\rangle w(a, k) q_{a, k}^{\varphi}\left(w\left(a^{\prime}, k^{\prime}\right)\right) ;
\end{aligned}
$$

here we use the fact that $\beta_{\gamma}$ leaves $v\left(\gamma^{\prime}\right)$ fixed. Hence $w$ realizes $\varphi$ with respect to $q^{\varphi}$.

Q. E. D。

Lemma 3.7. The action $q^{\varphi}$ is cocycle conjugate to $q$ for all $\varphi \in$ $Z^{1}(\mathscr{K}, \Gamma)$

Proof. Suppose that we can find $\left\{\sigma_{x}\right\}$ and $\{w(k)\}$ with

$$
\begin{aligned}
\sigma_{x} q_{a} & =q_{a} \sigma_{x}, \\
\sigma_{y}{ }^{\circ} q_{k} \circ \beta_{\varphi(k)}{ }^{\circ} \sigma_{x}^{-1} & =\operatorname{Ad}(w(k)) \circ q_{k}, k \in \mathscr{K}, k: x \rightarrow y, \\
\operatorname{Ad}(w(k)) q_{a} & =q_{a} \operatorname{Ad}(w(k)) .
\end{aligned}
$$

Then the hyperfiniteness of $\mathscr{K}$ enables us to adjust $w(k)$ into a cocycle with the above properties, which means that $q^{\varphi}$ and $q$ are cocycle conjugate as actions of $A \rtimes_{s} \mathscr{K}$.

Now, it follow from $q_{k} \cdot \beta_{\varphi(k)} \cdot q_{k}^{-1}=\beta_{\varphi(k) \cdot k^{-1}}$ that the actions: $k \mapsto q_{k} \cdot \beta_{\varphi(k)}$

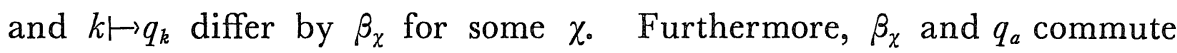
by construction, and

$$
\beta_{\chi}=\lim _{n \rightarrow \infty} \operatorname{Ad} b_{\chi, n}
$$

with

$$
b_{\chi, m}=\Pi_{n \leqq 0}^{\otimes} 1_{n} \otimes\left(\underset{1 \leqq n \leqq m}{\Pi_{1}} u_{n}(x, \chi)\right) \otimes \prod_{n>m}^{\otimes} 1_{n} \in \mathscr{Q}_{x} .
$$

Since we have

$$
q_{a}\left(b_{\chi, m}\right)=\langle a, \chi\rangle^{m} b_{\chi, m},
$$

$\operatorname{Ad}\left(b_{x, m}\right)$ and $q_{a}$ commute. Hence $\beta_{x}$ belongs to the group $C_{x}$ of all those $\theta \in \operatorname{Aut}\left(\mathscr{Q}_{x}\right)$ such that $\theta=\lim \operatorname{Ad}\left(b_{m}\right)$ for some $b_{m} \in \mathscr{Q}_{x}$ with $\operatorname{Ad}\left(b_{m}\right) \cdot q_{a}=q_{a} \cdot \operatorname{Ad}\left(b_{m}\right)$. By construction, we have

$$
C_{x}=\overline{\operatorname{Int}}_{q}\left(\mathscr{Q}_{x}\right) \text {, }
$$

where $\operatorname{Int}_{q}\left(\mathscr{Q}_{x}\right)$ is the group of all those inner automorphisms of $\mathscr{Q}_{x}$ which commute with $q(A(x))$. Thus, the Cohomology Lemma, [16, Theorem 5.5], yields the existence of $\left\{\sigma_{x}\right\} \subset C_{x}$ and $\{w(k)\}$ such that $\operatorname{Ad}(w(k)) \in \operatorname{Int}_{q}\left(\mathscr{Q}_{x}\right)$ and 


$$
\sigma_{y} \cdot q_{k} \cdot \beta_{\varphi(k)} \cdot \sigma_{x}^{-1}=\operatorname{Ad}(w(k)) q_{k}
$$

Q. E. D.

Thus we now conclude that

$$
\mathscr{C}(q)=Z^{1}(\mathscr{K}, \hat{A}),
$$

which in turn implies that there exists an action $p$ of $\mathscr{G}$ such that

$$
\begin{aligned}
& \mathscr{C}(p)=Z^{1}(\mathscr{K}, \widehat{\mathscr{H}}), \\
& \chi_{p}=1 \in \Lambda\left(\mathscr{G}, \mathscr{N}, \mathbb{T}^{\prime}\right) .
\end{aligned}
$$

Therefore, we have solved part (b) of the construction problem.

We now turn to the problem (a). Namely, we want an action $p$ of $\mathscr{G}$ on $\mathscr{R}$ such that

$$
\left\{\begin{array}{l}
p_{n}=\operatorname{Ad}(u(n)), n \in \mathscr{N} \\
u(m) u(n)=\mu(n, m) u(m n) \\
p_{r}\left(u\left(\gamma^{-1} n \gamma\right)\right)=\lambda(n, \gamma) u(n),(n, \gamma) \in(\mathscr{N} \times \mathscr{G}) \cap \mathscr{G}^{(2)}
\end{array}\right.
$$

Based on the semi-direct product $\mathscr{G}=\mathscr{H} \searrow_{s} \mathscr{H}$, we split the problem into the " $\mathscr{H}$-part" and the " $\mathscr{K}$-part". To put the " $\mathscr{H}$-part" and the " $\mathscr{K}$-part together, we observe the following: if $p_{g k}=\alpha_{g} \bullet \beta_{k}$, with $\alpha$ and $\beta$ actions of $\mathscr{H}$ and $\mathscr{K}$ respectively, then $p$ will be an action of $\mathscr{G}$ provided

$$
\alpha_{k h_{k}-1}=\beta_{k} \alpha_{h} \beta_{k}^{-1},(k, h) \in(\mathscr{K} \times \mathscr{H}) \cap \mathscr{G}^{(2)} .
$$

Next we have, with $\gamma=h k$,

$$
p_{r}\left(u\left(\gamma^{-1} n \gamma\right)\right)=p_{h k}\left(u\left(k^{-1} h^{-1} n h k\right)\right)=\alpha_{h} \cdot \beta_{k}\left(u\left(k^{-1} h^{-1} n h k\right)\right) ;
$$

and

$$
\lambda(n, \gamma) u(n)=\lambda(n, h k) u(n)=\lambda(n, h) \lambda\left(h^{-1} n h, k\right) u(n) .
$$

Thus, we shall need

$$
\alpha_{h} \beta_{k}\left(u\left(k^{-1} h^{-1} n h k\right)\right)=\lambda(n, h) \lambda\left(h^{-1} n h, k\right) u(n) ;
$$

and for this to obtain, it suffices to have

$$
\left\{\begin{array}{l}
\alpha_{h}\left(u\left(h^{-1} n h\right)\right)=\lambda(n, h) u(n),(n, h) \in(\mathscr{N} \times \mathscr{H}) \cap \mathscr{H}^{(2)}, \\
\beta_{k}\left(u\left(k^{-1} m k\right)\right)=\lambda(m, k) u(m),(m, k) \in(\mathscr{N} \times \mathscr{K}) \cap \mathscr{G}^{(2)} .
\end{array}\right.
$$

Now, $\mathscr{N}$ and $\mathscr{H}$ are nothing but Borel maps: $x \in X \rightarrow N_{x}$ and $x \in X \rightarrow H_{x}$ from $X$ to countable discrete amenable groups, with $N_{x} \triangleleft H_{x}$ for each $x$. So, we shall give a functorial construction which 
associates to each $H, N, \lambda$, $\mu$ with $N\left\langle H\right.$ and $(\lambda, \mu) \in Z\left(H, N, \mathbb{T}^{\urcorner}\right)$, a factor $\mathscr{R}_{H, N, \lambda, \mu}$ carrying an action $\alpha$ of $H$ for which $\chi_{\alpha}=[\lambda, \mu]$.

Given $\{H, N, \lambda, \mu\}$ as above, let

$$
\left\{\begin{array}{l}
\mathscr{R}_{H}=\prod_{n=1}^{\infty} \otimes \prod_{h \in H}^{\otimes} M(2, C)_{h, n}, \\
s_{h}\left(\prod_{n=1}^{\infty} \otimes\left(\prod_{h^{\prime} \in H} \otimes a_{h^{\prime}, n}\right)\right)=\prod_{n=1}^{\infty} \otimes\left(\underset{h^{\prime} \in H}{\prod^{\otimes} \otimes} a_{h h^{\prime}, n}\right) .
\end{array}\right.
$$

Namely, $s_{h}$ is the "Bernoulli" shift by $h \in H$. The infinite product is needed only when $H$ is finite to guarantee the freeness of the action s. Now, set

$$
\mathscr{R}(H, N, \lambda, \mu)=\mathscr{R}_{H} \rtimes_{s, \mu} N \text {. }
$$

This factor is generated by the following operators on the Hilbert space $\mathfrak{S}(H, N)=l^{2}(N) \otimes L^{2}\left(\mathscr{R}_{H}, \tau\right)$, where $\tau$ is the normalized trace on $\mathscr{R}_{H}$;

$$
\left\{\begin{array}{l}
(a \xi)(n)=s_{n}^{-1}(a) \xi(n), \quad a \in \mathscr{R}_{H}, \\
\left(u_{\mu}(m) \xi\right)(n)=\mu\left(n^{-1}, m\right) \xi\left(m^{-1} n\right), \quad m, n \in N
\end{array}\right.
$$

Lemma 3.8. The group $H$ acts on $\mathscr{R}(H, N, \lambda, \mu)$ as follows:

$$
\left\{\begin{array}{l}
\alpha_{h}(a)=s_{h}(a), \quad a \in \mathscr{R}(H), \\
\alpha_{h}\left(u_{\mu}\left(h^{-1} n h\right)\right)=\lambda(n, h) u_{\mu}(n), \quad h \in H, \quad n \in N .
\end{array}\right.
$$

The proof is routine, so we leave the details to the reader.

Suppose that $\theta:(N, H) \mapsto\left(N^{\prime}, H^{\prime}\right)$ is an isomorphism with $\theta^{*} \lambda=$ $\left(\delta_{1} \sigma\right)^{-1} \lambda^{\prime}$ and $\theta^{*} \mu=\left(\delta_{2} \sigma\right)^{-1} \mu^{\prime}$ 。 We define $\tilde{\beta}_{\theta}: \mathscr{R}_{H} \mapsto \mathscr{R}_{H^{\prime}}$ by:

$$
\tilde{\beta}_{\theta}\left(\prod_{n=1}^{\infty} \otimes\left(\prod_{h \in H}^{\otimes} a_{h, n}\right)\right)=\prod_{n=1}^{\infty} \otimes\left(\prod_{h \in N}^{\otimes} a_{\theta(h), n}\right),
$$

and $\beta_{\theta}: \mathscr{R}(H, N, \lambda, \mu) \mapsto \mathscr{R}\left(H^{\prime}, N^{\prime}, \lambda^{\prime}, \mu^{\prime}\right)$ by:

$$
\left\{\begin{array}{l}
\beta_{\theta}(a)=\tilde{\beta}_{\theta}(a), \quad a \in \mathscr{R}_{H} ; \\
\beta_{\theta}\left(u_{\mu}(n)\right)=\sigma(\theta(n)) u_{\mu^{\prime}}(\theta(n)), \quad n \in N .
\end{array}\right.
$$

One checks by straightforward calculations that $\beta_{\theta}$ is indeed an isomorphism.

Caution: In general, we do not have $\beta_{\theta_{1} \theta_{2}}=\beta_{\theta_{1}} \circ \beta_{\theta_{2}}$, since there will be no "multiplicative" choice of coboundaries, $\delta \sigma$. 
However, suppose $(\tilde{\lambda}, \tilde{\mu}) \in Z(\mathscr{G}, \mathcal{N}, \mathbb{T})$ with restriction $(\lambda, \mu) \in$ $Z(\mathscr{H}, \mathcal{N}, T)$. Then the arguments is $\S 2$ show that

$$
\left\{\begin{array}{c}
k^{*} \lambda=\lambda\left(\delta_{1} \sigma_{k}\right)^{-1} \\
\text { with } \sigma_{k}(n)=\tilde{\lambda}(n, k), \\
k^{*} \mu=\mu\left(\delta_{2} \sigma_{k}\right)^{-1}
\end{array}\right.
$$

and that

$$
\sigma_{k_{1} k_{2}}(n)=\sigma_{k_{1}}(n) \sigma_{k_{2}}\left(k_{1}^{-1} n k_{1}\right) .
$$

If $k \in \mathscr{K}, k: x \rightarrow y$, then we have a corresponding isomorphism

$$
\theta_{k}:\left(N_{x}, H_{x}\right) \mapsto\left(N_{y}, H_{y}\right) \text { given by } \theta_{k}(h)=k h k^{-1} \text {. }
$$

Let

$$
\beta_{k}: \mathscr{R}\left(H_{x}, N_{x}, \lambda_{x}, \mu_{x}\right) \mapsto \mathscr{R}\left(H_{y}, N_{y}, \lambda_{y}, \mu_{y}\right)
$$

be the corresponding isomorphism defined above.

Lemma 3.9. $p_{h k}=\alpha_{h} \beta_{k}$ defines an action of $\mathscr{G}$ on the field: $x \in X \mapsto$ $\mathscr{R}\left(H_{x}, N_{x}, \lambda_{x}, \mu_{x}\right)$ which realizes the invariant $(\lambda, \mu)$.

Proof. We have only to check that $\beta$ is an action of $\mathscr{K}$ satisfying (31). With $k \in \mathscr{K}, y \rightarrow z$, and $l \in \mathscr{K}, x \rightarrow y$, we have

$$
\begin{aligned}
& \beta_{k} \beta_{l}\left(\prod_{n \geqq 1}^{\otimes}\left(\prod_{h \in H_{x}}^{\otimes} a_{h, n}\right)\right)=\beta_{k}\left(\prod_{n \geqq 1} \otimes\left(\prod_{h \in H_{x}}^{\otimes} a_{l n l^{-1, n}}\right)\right) \\
& =\prod_{n \geqq 1}^{\otimes}\left(\prod_{h \in H_{x}}^{\otimes} a_{k l l l^{-1} k^{-1}, n}\right) \\
& =\beta_{k l}\left(\prod_{n \geqq 1}^{\otimes}\left(\prod_{h \in H_{x}}^{\otimes} a_{h, n}\right)\right) ; \\
& \begin{aligned}
\beta_{k} \beta_{l}(u(n)) & =\beta_{k}\left(\sigma_{l}\left(\ln l^{-1}\right) u\left(\ln l^{-1}\right)\right) \\
& =\sigma_{k}\left(k \ln l^{-1} k^{-1}\right) \sigma_{l}\left(\ln l^{-1}\right) u\left(k \ln l^{-1} k^{-1}\right) \\
& =\lambda\left(k \ln l^{-1} k^{-1}, k l\right) u\left(k \ln l^{-1} k^{-1}\right)=\beta_{k l}(u(n)) .
\end{aligned}
\end{aligned}
$$

The above calculation shows that

$$
\beta_{k}(u(n))=\sigma_{k}\left(k n k^{-1}\right) u\left(k n k^{-1}\right),
$$

or

$$
\beta_{k}\left(u\left(k^{-1} n k\right)\right)=\lambda(n, k) u(n) .
$$

We now check $\alpha_{k h k}{ }^{-1} \beta_{k}=\beta_{k} \alpha_{k}$. To this end, we check only for $u(n)$ 's, since the other part is a triviality as we have observed above: 


$$
\begin{aligned}
& \begin{aligned}
\alpha_{k h k^{-1}} \beta_{k}\left(u\left(k^{-1} n k\right)\right) & =\alpha_{k h k^{-1}}(\lambda(n, k) u(n)) \\
= & \lambda(n, k) \lambda\left(k h k^{-1} n k h^{-1} k^{-1}, k h k^{-1}\right) u\left(k h k^{-1} n k h^{-1} k^{-1}\right)
\end{aligned} \\
& \begin{aligned}
\beta_{k} \alpha_{h}\left(u\left(k^{-1} n k\right)\right)= & \beta_{k}\left(\lambda\left(h k^{-1} n k h, h\right) u\left(h k^{-1} n k h^{-1}\right)\right) \\
= & \lambda\left(h k^{-1} n k h^{-1}, h\right) \lambda\left(k h k^{-1} n k h^{-1} k^{-1}, k\right) u\left(k h k^{-1} n k h^{-1} k^{-1}\right)
\end{aligned}
\end{aligned}
$$

Thus, we further compute

$$
\begin{aligned}
\lambda(n, k) \lambda( & \left.k h k^{-1} n k h^{-1} k^{-1}, k h k^{-1}\right) \\
& =\lambda(n, k) \lambda\left(k h k^{-1} n k h^{-1} k^{-1}, k\right) \lambda\left(h k^{-1} n k h^{-1}, h k^{-1}\right) \\
& =\lambda\left(k h k^{-1} n k h^{-1} k^{-1}, k\right) \lambda(n, k) \lambda\left(h k^{-1} n k h^{-1}, h\right) \lambda\left(k^{-1} n k, k^{-1}\right) \\
& =\lambda\left(k h k^{-1} n k h^{-1} k^{-1}, k\right) \lambda\left(h k^{-1} n k h^{-1}, h\right)
\end{aligned}
$$

because

$$
\lambda(n, k) \lambda\left(k^{-1} n k, k^{-1}\right)=\lambda\left(n, k k^{-1}\right)=1 .
$$

Thus we complete the proof.

Q. E.D.

Therefore, we have completed the "(a)-part" of Theorem 3.1. Together with the previous "(b)-part", we complete the proof of Theorem 3.1.

We shall denote this action of $\mathscr{G}$ by $m$ and call it the model action with $\chi_{m}=[\lambda, \mu] \in \Lambda(\mathscr{G}, \mathscr{N}, T)$.

\section{§4. Proof of Theorem 1.2-Comparison with the Model}

We complete the proof of Theorem 1.2 by showing that a given action $\alpha$ of $\mathscr{G}$ is cocycle conjugate to the model action $m$ with the same invariants. So we fix an action $\alpha$ of $\mathscr{G}$ on $\mathscr{P}$.

Note that $h \in \mathscr{H} \mapsto \alpha_{h}, m_{h}$ are both Borel fields of actions of the isotropy groups with the same (field of) characteristic invariants. By a result of Ocneanu, [14], we can choose $\left\{\theta_{x}: x \in X\right\}$ and $\{v(h)\}$ with the properties:

$$
\begin{aligned}
& \theta_{x} \circ \alpha_{h} \circ \theta_{x}^{-1}=\operatorname{Ad} v(h) \circ m_{h} \text { on } \mathscr{H}, \\
& v \in Z_{m}^{1}(\mathscr{H}, \mathscr{U}(\mathscr{P}))
\end{aligned}
$$

Here, $Z_{m}^{1}(\mathscr{H}, \mathscr{U}(\mathscr{P}))$ is the space of $m$-cocycles with values in the unitary group $\mathscr{U}(\mathscr{P})$.

Replacing $\alpha$ by $\gamma \in \mathscr{G} \rightarrow \theta_{r(\gamma)}{ }^{\circ} \alpha_{\gamma^{\circ}} \theta_{s(\gamma)}^{-1}$, we may suppose that

a) $\alpha$ and $m$ have the same invariants,

b) $\alpha_{h}=\operatorname{Ad} v(h) \circ m_{h}$ on $\mathscr{H}$. 
Now define $\beta_{k}$ by the relation

$$
\alpha_{k}=\beta_{k} \cdot m_{k}, \quad k \in \mathscr{K} .
$$

Lemma 4.1. We have

$$
\beta_{k} \cdot m_{k} \bullet \beta_{k}^{-1}=\operatorname{Ad} w(h, k) \cdot m_{k},(h, k) \in(\mathscr{H} \times \mathscr{K}) \cap \mathscr{G}^{(2)},
$$

where

$$
w(h, k)=\alpha_{k}\left(v\left(k^{-1} h k\right) *\right) v(h),
$$

and

$$
w\left(h h^{\prime}, k\right)=w(h, k) m_{h}\left(w\left(h^{\prime}, k\right)\right) .
$$

The proof is a straight forward computation and left to the reader.

Let $\mathscr{Q}_{x}=\mathscr{P} \rtimes_{m} H_{x}$ for each $x$, and let $\hat{m}_{x}$ denote the coaction of $H_{x}$ on $\mathscr{Q}_{x}$ dual to $m$, [13]. We set

$$
\mathscr{A}(x)=\left\{\sigma \in \operatorname{Aut}\left(\mathscr{Q}_{x}\right):(\sigma \otimes \iota) \cdot \hat{m}_{x}=\hat{m}_{x} \cdot \sigma\right\} ;
$$

Note that each $\sigma \in \mathscr{A}(x)$ is determined uniquely by its restriction $\theta$ to $\mathscr{P} \subseteq \mathscr{Q}_{x}$, and a $w \in Z_{m}^{1}\left(H_{x}, \mathscr{U}(\mathscr{P})\right)$ satisfying

$$
\theta \cdot m_{h} \bullet \theta^{-1}=\operatorname{Ad} w(h) \cdot m_{h} \quad \text { on } H_{x} \text {. }
$$

According to convenience, we shall think of elements of $\mathscr{A}(x)$ either as automorphisms of $\mathscr{Q}_{x}$, or as pairs $(\theta,\{w(h)\})$ satisfying (8) with $w \in Z_{m}^{1}\left(H_{x}, \mathscr{U}(\mathscr{P})\right)$ 。

Also, each $\mathscr{A}(x)$ is a Polish subgroup of $\operatorname{Aut}\left(\mathscr{Q}_{x}\right)$ and since the $\mathscr{Q}_{x}$ are isomorphic to a fixed algebra, $x \mapsto \mathscr{A}(x)$ is a Borel map as in [16; Theorem 2.3]. Further, if $k \in \mathscr{K}, k: x \rightarrow v$ and $\tilde{m}_{k}$ is the natural extension of $m_{k}$ to an isomorphism $\mathscr{Q}_{x} \mapsto \mathscr{Q}_{y}, \tilde{m}_{k} \mathscr{A}(x) \tilde{m}_{k}^{-1}=\mathscr{A}(y)$ so that $x \mapsto \mathscr{A}(x)$ may be viewed as a Borel functor to polish groups as in $[16 ; \S 4]$.

For $n \in \mathscr{N}$, let $u(n) \in \mathscr{U}(\mathscr{P})$ be unitaries with

$$
\left\{\begin{array}{l}
m_{n}=\operatorname{Ad} u(n), \quad \text { on } \mathscr{N}=\mathscr{N}_{\alpha}=\mathscr{N}_{m}, \\
u(n) u\left(n^{\prime}\right)=\mu\left(n, n^{\prime}\right) u\left(n n^{\prime}\right) \quad \text { on } \mathscr{N}^{(2)} .
\end{array}\right.
$$

If $\sigma=(\theta,\{w(h)\}) \in \mathscr{A}(x)$, then

$$
\text { Ad } \theta(u(n))=\theta \cdot m_{n} \cdot \theta^{-1}=\operatorname{Ad} w(n) u(n),
$$

so

$$
\theta(u(n))=c_{\sigma}(n) w(n) u(n)
$$


for some $c_{\sigma}(n) \in \mathbb{T}$. Routine calculations show that

$$
\left\{\begin{array}{l}
c_{\sigma}\left(n n^{\prime}\right)=c_{\sigma}(n) c_{\sigma}\left(n^{\prime}\right), \text { and } \\
c_{\sigma \cdot \sigma^{\prime}}(n)=c_{\sigma}(n) c_{\sigma^{\prime}}(n)
\end{array}\right.
$$

We define

$$
\mathscr{A}^{0}(x)=\left\{\sigma \in \mathscr{A}(x): c_{\sigma}=1 \text { on } N_{x}\right\},
$$

and let $\sigma_{k} \in \mathscr{A}(x)$ be the element determined by $\left(\beta_{k},\{w(k, h)\}\right)$ via equations (4) and (5).

\section{Lemma 4.2。}

a) $\left\{\mathscr{A}^{0}(x)\right\}$ is $\mathscr{K}$-invariant, and $\dot{\cup} \mathscr{A}^{0}(x) \subseteq \dot{U} \mathscr{A}(x)$ is Borel;

b) $\sigma_{k} \in \mathscr{A}^{0}(r(k))$ for $k \in \mathscr{K}$.

Proof. a) Let $(\theta,\{w(h)\}) \in \mathscr{A}^{0}(x)$; note that

$$
\begin{aligned}
& m_{k} \circ \theta \cdot m_{k}^{-1} \cdot m_{h} \circ m_{k} \circ \theta^{-1} \cdot m_{k}^{-1}=m_{k} \circ \theta \circ m_{k^{-1} h k} \circ \theta^{-1} \cdot m_{k}^{-1} \\
& \quad=m_{k} \circ \operatorname{Ad} w\left(k^{-1} h k\right) \cdot m_{k}-1 \cdot{ }_{h k} \cdot m_{k}^{-1}=\operatorname{Ad~} m_{k}\left(w\left(k^{-1} h k\right)\right) \circ m_{h},
\end{aligned}
$$

and

$$
\begin{aligned}
m_{k} \circ \theta \cdot m_{k}^{-1}(u(n)) & =m_{k} \cdot \theta\left(\lambda(n, k)^{-1} u\left(k^{-1} n k\right)\right) \\
& =\lambda(n, k)^{-1} m_{k}\left(w\left(k^{-1} n k\right) u\left(k^{-1} n k\right)\right) \\
& =m_{k}\left(w\left(k^{-1} n k\right)\right) u(n),
\end{aligned}
$$

where $\lambda$ is (part of) the characteristic invariant of $m$. Thus $\left(m_{k} \circ \theta^{\circ} m_{k}^{-1}\right.$, $\left\{m_{k}\left(w\left(k^{-1} h k\right)\right\} \in \mathscr{A}^{0}(r(k))\right.$ as claimed.

With $v$ as in equation (1), and $u(n)$ as in equation (9), let

$$
a(n)=v(n) u(n), \quad n \in \mathcal{N},
$$

so

$$
\alpha_{n}=\operatorname{Ad} a(n) .
$$

Now we may suppose that $\lambda_{m}=\lambda_{\alpha}$ on $(\mathscr{N} \times \mathscr{G}) \cap \mathscr{G}^{(2)}$, so

$$
\begin{aligned}
\beta_{k}(u(n)) & =\alpha_{k} \circ m_{k}^{-1}(u(n))=\lambda_{m}(n, k)^{-1} \alpha_{k}\left(u\left(k^{-1} n k\right)\right) \\
& =\lambda_{\alpha}(n, k)^{-1} \alpha_{k}\left(v\left(k^{-1} n k\right)^{*} a\left(k^{-1} n k\right)\right)=\alpha_{k}\left(v\left(k^{-1} n k\right)^{*}\right) a(n) \\
& =\alpha_{k}\left(v\left(k^{-1} n k\right) *\right) v(n) u(n)=w(n, k) u(n),
\end{aligned}
$$

from which we conclude $\sigma_{k} \in \mathscr{A}^{0}(x)$.

It is clear from the definition of $\mathscr{A}^{0}(x)$, equation (11), that it is a closed subgroup of $\mathscr{A}(x)$, and that $\dot{\cup} \mathscr{A}^{0}(x)$ is Borel in $\dot{U} \mathscr{A}(x)$. 
Note that since both $m_{k}$ and $\alpha_{k}$ may be assumed to scale the trace $\tau$ on $\mathscr{P}$ by the same factor (automatically one unless $\mathscr{P}$ is of type $\left.\mathbb{I I}_{\infty}\right), \sigma_{k} \in \mathscr{A}^{1}(r(k))$, where

$$
\mathscr{A}^{1}(x)=\mathscr{A}^{0}(x) \cap\left\{\sigma \in \operatorname{Aut}\left(\mathscr{Q}_{x}\right) ; \hat{\tau}_{x} \sigma=\hat{\tau}_{x}\right\},
$$

$\tau_{x}$ being the trace on $\mathscr{Q}_{x}$ dual to $\tau$ on $\mathscr{P}$, as in [19].

Lemma 4. 3. For $a \in \mathscr{U}(\mathscr{P})$, let $\pi_{x}(a)=\left(\operatorname{Ad} a,\left\{a m_{h}\left(a^{*}\right)\right\}\right)$ for $h \in H_{x}$. Then $\mathscr{B}: x \in X \mapsto \mathscr{B}(x)=\pi_{x}(\mathscr{U}(\mathscr{P}))$ is a dense, normal Borel subfunctor of $\mathscr{A}^{1}$ i.e. $\mathscr{B}(x)$ is Borel and normal in $\mathscr{A}^{1}(x), \mathscr{B} \triangleleft \mathscr{A}^{1}$ as in $[16 ;$ Definition 5.3] and $\mathscr{B}(x) \subseteq \mathscr{A}^{1}(x)$ is dense for each $x \in X$.

Proof. The fact that $\mathscr{B}(x) \subseteq \mathscr{A}^{1}(x)$ is Borel and normal follows as in the proof of Lemma 2.5.6 of [10]; the invariance of $\mathscr{A}^{1}$ and $\mathscr{B}$ under the action of $\mathscr{K}$ is obvious. Since the map

$$
\pi: \quad(x, \dot{a}) \in X \times \mathscr{U}(\mathscr{P}) / T \mapsto \pi_{x}(a) \in \mathscr{B}(x)
$$

is surjective to $\dot{\cup} \mathscr{B}(x)$, is continuous in $\dot{a}$ for fixed $x$ and Borel in $x$ for fixed $\dot{a} \in \mathscr{U}(\mathscr{P}) / \mathbb{T}, \pi$ is a Borel isomorphism so that $\dot{\cup} \mathscr{B}(x) \subseteq$ $\dot{U} \mathscr{A}^{1}(x)$ is a Borel set.

It remains to prove that $\mathscr{B}(x)$ is dense in $\mathscr{A}^{1}(x)$ for each $x$. The proof is modelled on that of Lemma 2.5.6 of [10]. So let $\sigma=$ $(\theta,\{w(h)\}) \in \mathscr{A}^{1}(x)$; with $u(n)$ as in equation (9), we have, by definition,

$$
\theta(u(n))=w(n) u(n) .
$$

Equation (12) replaces Equation 2.5.7 of the proof of Lemma 2.5.6 of [10], and the our Lemma follows in exactly the same way as in [10] - the hypothesis that the group be abelian in [10] is not used except to reach equation 2.5.6.

Q. E. D.

We may now apply the Cohomology Lemma, [16, Theorem 5.5] to $\mathscr{B}$ and $\mathscr{A}^{1}$, and the $m$-cocycle $\beta_{k}$; we conclude the existence of a Borel map $x \in X \rightarrow \sigma_{x}=\left(\theta_{x},\left\{w_{x}(h)\right\}\right) \in \mathscr{A}^{1}(x)$, and inner automorphisms Ad $a(k), k \in \mathscr{K}$, with

$$
\theta_{y} \cdot \beta_{k} \cdot m_{k} \cdot \theta_{x}^{-1} \cdot m_{k}^{-1}=\operatorname{Ad} a(k)
$$

for $k: x \rightarrow y$ in $\mathscr{K}$; since $\beta_{k}=\alpha_{k} \cdot m_{k}^{-1}$, this yields

$$
\theta_{y} \cdot \alpha_{k} \cdot \theta_{x}^{-1}=\operatorname{Ad} a(k) \cdot m_{k}
$$


Since $\mathscr{K}$ is hyperfinite, we may assume $a \in Z_{m}^{1}(\mathscr{K}, \mathscr{U}(\mathscr{P}))$

Now, for arbitrary $\gamma=h k \in \mathscr{G}$, we have

$$
\begin{aligned}
\theta_{y} \circ \alpha_{h k} \circ \theta_{x}^{-1} & =\theta_{y} \circ \alpha_{h} \circ \theta_{y}^{-1} \circ \theta_{y} \circ \alpha_{k} \circ \theta_{x}^{-1} \\
& =\theta_{y} \circ \operatorname{Ad} v(h) \cdot m_{h} \circ \theta_{y}^{-1} \circ \text { Ad } a(k) \circ m_{k} \\
& =\operatorname{Ad} \theta_{y}(v(h)) w_{y}(h) \circ m_{h} \circ \text { Ad } a(k) \circ m_{k} \\
& =\operatorname{Ad~} \theta_{y}(v(h)) w_{y}(h) m_{h}(a(k)) \circ m_{h k},
\end{aligned}
$$

so that for all $\gamma \in \mathscr{G}, \theta_{y} \circ \alpha_{\gamma} \theta_{x}^{-1}=m_{\gamma} \bmod \operatorname{Int}(\mathscr{P})$.

We replace $\alpha_{r}$ by $\theta_{y} \circ \alpha_{r} \theta_{x}^{-1}$, and define

$$
b(h k)=\theta_{y}(v(h)) w_{y}(h) m_{h}(a(k)), h \in H_{y},
$$

so that we have now

$$
\alpha_{h k}=\operatorname{Ad} b(h k) \circ m_{h k}
$$

A routine calculation shows that $h \in \mathscr{H} \mapsto b(h)=\theta_{y}(v(h)) w_{y}(h)$ defines an element of $Z_{m}^{1}(\mathscr{H}, \mathscr{U}(\mathscr{P}))$; we already know that $k \in \mathscr{K} \rightarrow b(k)=a(k)$ defines an element of $Z_{m}^{1}(\mathscr{K}, \mathscr{U}(\mathscr{P}))$.

Lemma 4. 4. With notation as above, and $g, h \in \mathscr{H}$ and $k, l \in \mathscr{K}$,

$$
b(g k) m_{g k}(b(h l))=\varphi(k, h) b(g k h l)
$$

for some $\varphi \in Z^{1}(\mathscr{K}, \widehat{\mathscr{H}})$.

Proof. Since $\alpha_{r}=\operatorname{Ad} b(\gamma) \cdot m_{r}$ on $\mathscr{G}$, we have

$$
b(g k) m_{g k}(b(h l))=\kappa(g k, h l) b(g k h l)
$$

for some $\kappa \in Z^{2}(\mathscr{G}, \mathbb{T})$. However, we also have

$$
b(g k) m_{g k}(b(h l))=b(g) m_{g}\left(b(k) m_{k}\left(b(h) m_{h}(b(l))\right)\right),
$$

while

$$
\begin{aligned}
b(g k h l) & =b\left(g k h k^{-1} k l\right)=b\left(g k h k^{-1}\right) m_{g k h k^{-1}}(b(k l)) \\
& =b(g) m_{g}\left(b\left(k h k^{-1}\right) m_{k h k^{-1}}\left(b(k) m_{k}(b(l))\right)\right) \\
& =b(g) m_{g}\left(b\left(k h k^{-1}\right) m_{k}\left(m_{h^{-1}}\left(b(k) m_{k}(b(l))\right)\right) .\right.
\end{aligned}
$$

Comparing equations (15) and (16) we see that $\kappa(g k, h l)$ depends only on the middle two variables, $k$ and $h$, so that

$$
\kappa(g k, h l)=\varphi(k, h)
$$

for some Borel function $\varphi$ on $(\mathscr{K} \times \mathscr{H}) \cap \mathscr{G}^{(2)}$. A routine calculation using the fact that $\kappa \in Z^{2}(\mathscr{G}, \mathbb{T})$ shows that $\varphi \in Z^{1}(\mathscr{K}, \widehat{\mathscr{H}})$. Q. E. D. 
We now recall that the model action $m$ was constructed, at considerable effort, so that $\mathscr{C}(m)=Z^{1}(\mathscr{H}, \mathscr{H})$. Thus we can choose automorphisms $\left\{\theta_{x}\right\}$ of $\mathscr{P}$ and unitaries $\{c(\gamma): \gamma \in \mathscr{G}\}$ in $\mathscr{P}$ such that

$$
\left\{\begin{array}{l}
\theta_{y} \cdot m_{r} \cdot \theta_{x}^{-1}=\operatorname{Ad} c(\gamma) \cdot m_{\gamma} \text { on } \mathscr{G}, \\
c(g k) m_{g k}(c(h l))=\varphi \overline{(k, h)} c(g k h l), \\
h \in \mathscr{H} \rightarrow c(h) \text { in } Z_{m}^{1}(\mathscr{H}, \mathscr{U}(\mathscr{P})), \\
k \in \mathscr{K} \rightarrow c(k) \text { in } Z_{m}^{1}(\mathscr{H}, \mathscr{U}(\mathscr{P}))
\end{array}\right.
$$

Completion of Proof of Theorem 1.2. With $\alpha$ as in equation (14) and $\theta$ and $c$ as in equation (17), we have

$$
\theta_{y} \cdot \alpha_{\gamma} \cdot \theta_{x}^{-1}=\operatorname{Ad} \theta_{y}(b(\gamma)) c(\gamma) \cdot m_{\gamma},
$$

for $r \in \mathscr{G}, r(\gamma)=y, s(\gamma)=x$. With $g, h \in \mathscr{H}$ and $k, l \in \mathscr{K}$ where $l: x \rightarrow y$ and $k: y \rightarrow z$, and $\gamma=g k, \gamma^{\prime}=h l$, we have

$$
\begin{aligned}
\theta_{z}(b(\gamma)) c & (\gamma) m_{\gamma}\left(\theta_{y}\left(b\left(\gamma^{\prime}\right)\right) c\left(\gamma^{\prime}\right)\right) \\
& =\theta_{z}(b(\gamma)) \theta_{z} \circ m_{r}\left(b\left(\gamma^{\prime}\right)\right) c(\gamma) m_{r}\left(c\left(\gamma^{\prime}\right)\right) \\
& =\theta_{z}\left(\varphi(k, h) b\left(\gamma \gamma^{\prime}\right)\right) \varphi(k, h) c\left(\gamma \gamma^{\prime}\right)=\theta_{z}\left(b\left(\gamma \gamma^{\prime}\right)\right) c\left(\gamma \gamma^{\prime}\right),
\end{aligned}
$$

so that $\gamma \mapsto \theta_{r(\gamma)}(b(\gamma)) c(\gamma)$ falls in $Z_{m}^{1}(\mathscr{G}, \mathscr{U}(\mathscr{P}))$, and $\alpha$ is cocycle conjugate to $m$ as required.

Q.E. D.

\section{§5. Applications}

Our first application is to the existence of Cartan subalgebras, in the sense of [7], which are invariant under the action of a group or groupoid. If $\alpha$ is an action of an orbitally discrete measured groupoid $(\mathscr{G}, \nu)$ on a factor $\mathscr{P}$, an $\alpha$-invariant Cartan subalgebra of $\mathscr{P}$ will mean a Borel family $\left\{\mathscr{D}_{x}: x \in \mathscr{G}^{\left({ }^{0}\right)}\right\}$ of Cartan subalgebras of $\mathscr{P}$ such that $\alpha_{r}\left(\mathscr{D}_{s(r)}\right)=\mathscr{D}_{r(r)}$ a. e. on $\mathscr{G}$.

Theorem 5.1. Let $\alpha$ be an action of a discrete amenable group $G$ on a semifinite injective von Neumann algebra $\mathscr{M}$, or of an orbitally discrete amenable measured groupoid $(\mathscr{G}, \nu)$ on a semifinite injective factor $\mathscr{P}$. Then $\alpha$ is concycle conjugate to an action which possesses an invariant Cartan subalgebra.

Proof. Consider first the case of actions of groupoids. By Theorem 1.2, it suffices to show that each of the model actions constructed in 
$\S 3$ admits an invariant Cartan subalgebra-in fact, we do not have to worry about part " $b$ " of the construction of the model either.

Note that each of the factors $\mathscr{R}_{H}$ ( $\$ 3$, equation 33 ) has a Cartan subalgebra $\mathscr{D}_{H}=\prod_{n=1}^{\infty} \otimes \prod_{h \in H}^{\otimes} D(2, \mathbb{C})_{n, h}$, where $D(2, \mathbb{C}) \subseteq M(2, \mathbb{C})$ is the diagonal matrices. Clearly, $\mathscr{D}_{H}$ is globally invariant under the shift $s_{h}, h \in H$, so that its image $\mathscr{D}(H, N, \lambda, \mu)$ in $\mathscr{R}(H, N, \lambda, \mu)=\mathscr{R}_{H} \rtimes_{s, \mu} H$ is again a Cartan subalgebra; further $\mathscr{D}(H, N, \lambda, \mu)$ is manifestly invariant under the action of $H$ defined in Lemma 3.8. It is also clear that if $\theta:(H, N, \lambda, \mu) \mapsto\left(H^{\prime}, N^{\prime}, \lambda^{\prime}, \mu^{\prime}\right)$ is as in the discussion following Lemma 3.8 , the corresponding isomorphism $\beta_{\theta}: \mathscr{R}(H, N, \lambda, \mu)$ $\rightarrow \mathscr{R}\left(H^{\prime}, N^{\prime}, \lambda^{\prime}, \mu^{\prime}\right)$ maps $\mathscr{D}(H, N, \lambda, \mu)$ to $\mathscr{D}\left(H^{\prime}, N^{\prime}, \lambda^{\prime}, \mu^{\prime}\right)$. Thus if $\mathscr{P}=\mathscr{R}$ is the hyperfinite $\mathrm{II}_{1}$-factor, and $\alpha$ has characteristic invariant $\chi=[\lambda, \mu]$, the Cartan subalgebras $\mathscr{D}\left(H_{x}, N_{x}, \lambda_{x}, \mu_{x}\right)$ are coherent under the model action with the same invariants.

Clearly the reductions to the case $\delta=1$, and subsequently to the $\mathrm{II}_{1}$-case, accomplished in observations 1) and 2) at the beginning of the proof of Theorem 3.1 permit the synthesis of a suitable family of Cartan subalgebras for any model action of $(\mathscr{G}, 2)$.

In the case of actions of groups, we let $(\mathscr{G}, \nu)$ be the ancillary groupoid with action $\alpha$ on $\mathscr{P}$; if $\left\{\mathscr{D}_{x}: x \in \mathscr{G}^{(0)}\right\}$ is invariant under an action $m$ of $(\mathscr{G}, \nu)$ cocycle conjugate to $\alpha$, then $\int^{\oplus} \mathscr{D}_{x} d \nu(x)$ is a Cartan subalgebra of $L^{\infty}(X, \nu ; \mathscr{P})$ invariant under an action of $G$ cocycle conjugate to the given one.

Q.E.D.

This result in turn has applications to the structure of group von Neumann algebras of non-simple discrete amenable groups; the first named author intends to present these elsewhere.

Our second application is to the classification of coactions of discrete amenable groups $G$ on semifinite injective von Neumann algebras $\mathscr{M}$; for the notion of coaction, we refer the reader to [13], whose notation we shall use.

Definition 5.2. Let $\delta_{1}, \delta_{2}$ be coactions of $G$ on $\mathscr{M}_{1}$ and $\mathscr{M}_{2}$ respectively.

a) $\delta_{1}$ and $\delta_{2}$ are conjugate if, for some isomorphism $\theta: \mathscr{M}_{1} \rightarrow \mathscr{M}_{2}$ we have $(\theta \otimes \iota) \circ \delta_{1}=\delta_{2} \circ \theta$; 
b) $\delta_{1}$ and $\delta_{2}$ are stably conjugate if $\bar{\delta}_{1}$ is conjugate to $\bar{\delta}_{2}$. Here $\bar{\delta}_{j}$ is the coaction defined in [13; Theorem 2.7] by

$$
\bar{\delta}_{j}(x)=\operatorname{Ad}\left(1 \otimes W_{G}\right) \cdot(\iota \otimes \sigma) \cdot\left(\delta_{j} \otimes \iota\right)(x)
$$

for $x \in \mathscr{M}_{j} \otimes \mathscr{Q} \mathscr{L}\left(L^{2}(G)\right)$. Note that $\overline{\boldsymbol{\delta}}_{j}$ is conjugate to the second dual coaction $\hat{\delta}_{j}$ by [13; Theorem 2.7], so that stable conjugacy may also be interpreted in terms of $\hat{\delta}_{1}$ and $\hat{\delta}_{2}$.

If $\delta$ is a coaction of $G$ on $\mathscr{M}$, we let $\delta$ denote the dual action of $G$ on $\mathscr{M} \rtimes_{\delta} G$, [13; Proposition 2.4]. The next result is then a special case of [23; Proposition 2.1]. Since the result of Nakagami is presented in the context of Kac algebras, we present the full proof for the sake of the reader's convenience.

Theorem 5.3. Let $\delta_{1}$ and $\delta_{2}$ be coactions of an arbitrary second countable locally compact group $G$ on von Neumann algebras $\mathscr{M}_{1}$ and $\mathscr{M}_{2 .}$ Then $\delta_{1}$ is stably conjugate to $\delta_{2}$ if and only if $\hat{\delta}_{1}$ is cocycle conjugate to $\hat{\delta}_{2}$.

Proof. Let $\mathscr{N}_{j}=\mathscr{M}_{j} \rtimes_{\delta_{j}} G$ and suppose that $\hat{\delta}_{1}$ is conjugate to $\hat{\delta}_{2}$ via an isomorphism $\varphi: \mathscr{N}_{1} \searrow_{\hat{\delta}_{1}} G \rightarrow \mathscr{N}_{2} \searrow_{\hat{\delta}_{2}} G$. Note that if $x \in \mathscr{N}_{1} \searrow_{\hat{\delta}_{1}} G$, then $\hat{\delta}_{1}(x)=x \otimes 1$ if and only if $\hat{\delta}_{2}(\varphi(x))=\varphi(x) \otimes 1$. Thus by [13; Chapter II, Theorem 1. 1], $\varphi$ restricts to an isomorphism $\theta$ of $\mathscr{N}_{1} \subseteq \mathscr{N}_{1} \rtimes_{\hat{\delta}_{1}} G$ to $\mathscr{N}_{2}$.

Further, if $\rho$ is the right regular representation of $G$ on $L^{2}(G)$, and we define $u(r)$ for $r \in G$ by the relation

$$
\varphi(1 \otimes 1 \otimes \rho(r))=u(r)(1 \otimes 1 \otimes \rho(r)),
$$

then since

$$
\hat{\delta}_{2}(u(r)(1 \otimes 1 \otimes \rho(r)))=\hat{\delta}_{2}(u(r))(1 \otimes 1 \otimes \rho(r) \otimes \rho(r)),
$$

and

$$
\begin{aligned}
\hat{\delta}_{2} \cdot \varphi(1 \otimes 1 \otimes \rho(r)) & =(\varphi \otimes \iota)(1 \otimes 1 \otimes \rho(r) \otimes \rho(r)) \\
& =(u(r) \otimes 1)(1 \otimes 1 \otimes \rho(r) \otimes \rho(r)),
\end{aligned}
$$

we see $\hat{\delta}_{2}(u(r))=u(r) \otimes 1$, and $u(r) \in \mathscr{N}_{2}$.

Applying $\varphi$ to the identity

$$
(1 \otimes 1 \otimes \rho(r)) \quad(1 \otimes 1 \otimes \rho(s))=(1 \otimes 1 \otimes \rho(r s)),
$$

we find

$$
u(r s)=u(r) \quad \operatorname{Ad}(1 \otimes 1 \otimes \rho(r))(u(s))
$$


since $\operatorname{Ad}(1 \otimes 1 \otimes \rho(r))=\hat{\delta}_{2, r}$ on $\mathscr{N}_{2} \subseteq \mathscr{N}_{2} \rtimes_{\hat{\delta}_{2}} G, r \mapsto u(r)$ is a 1 -cocycle for the action $\hat{\delta}_{2}$ of $G$ on $\mathscr{N}_{2}$. Finally

$$
\begin{aligned}
\varphi \cdot \operatorname{Ad}(1 \otimes 1 \otimes \rho(r)) \cdot \varphi^{-1} & =\operatorname{Ad} \varphi(1 \otimes 1 \otimes \rho(r)) \\
& =\operatorname{Ad} u(r) \cdot \operatorname{Ad}(1 \otimes 1 \otimes \rho(r)),
\end{aligned}
$$

so that $\theta \cdot \hat{\delta}_{1, r} \cdot \theta^{-1}=\operatorname{Ad} u(r) \cdot \hat{\delta}_{2, r}$ as required.

Conversely, if $\theta: \mathscr{N}_{1} \rightarrow \mathscr{N}_{2}$ is an isomorphism with

$$
\theta \cdot \hat{\delta}_{1, r} \theta^{-1}=\operatorname{Ad} u(r) \cdot \hat{\delta}_{2, r}
$$

for some $\hat{\delta}_{2}$-cocycle $\{u(r)\}$, we may suppose that $\theta$ is implemented by a unitary $U: \mathfrak{S}_{1} \rightarrow \mathscr{S}_{2}$ (where $\mathscr{M}_{j}$ acts on $\mathscr{S}_{j}$ ), and define $V: L^{2}\left(G, \mathfrak{S}_{1}\right)$ $\mapsto L^{2}\left(G, \mathfrak{S}_{2}\right)$ by

$$
(V \xi)(r)=u(r) * U \xi(r), \xi \in L^{2}\left(G, \mathfrak{S}_{1}\right) \text {. }
$$

A routine calculation shows that $V$ implements an isomorphism of $\mathscr{N}_{1} \rtimes_{\hat{\delta}_{1}} G$ with $\mathscr{N}_{2} \rtimes_{\hat{\delta}_{2}} G$ with (Ad $\left.V \otimes \iota\right) \circ \hat{\delta}_{1}=\hat{\delta}_{2} \cdot$ Ad $V$, so $\delta_{1}$ is stably conjugate to $\delta_{2}$.

Q.E.D.

We now turn to the question of conjugacy of coactions. Recall that if $\delta$ is a coaction of $G$ on $\mathscr{M}$, and if $p \in \mathscr{M}$ is a $\delta$-invariant projection, then $\delta_{p}: x \in p \mathscr{M} p \mapsto \delta(x) \in p \mathscr{M} p \bar{\otimes} \mathscr{R}(G)$ is again a coaction.

Lemma 5.4. Suppose $\delta$ is a coaction of the discrete group $G$ on $\mathscr{M}$, and $p=1 \otimes e$, where $e \in \mathscr{L}\left(L^{2}(G)\right)$ is the projection on the subspace spanned by the characteristic function of the identity. Then $p$ is $\bar{\delta}$-invariant and $\bar{\delta}_{p}$ is conjugate to $\delta$.

Proof. The invariance $p$ under $\bar{\delta}$ is a special case of Equation 2.23 of $[13$, Chapter I]. To establish the conjugacy, define $\theta: \mathscr{M} \rightarrow$ $p\left(\mathscr{M} \otimes \mathscr{L}\left(L^{2}(G)\right)\right) p$ by $\theta(x)=x \otimes e$; note that for $a \in \mathscr{M}, r \in G$ and $\xi \in L^{2}(G \times G ; \mathfrak{L})$,

$$
\begin{aligned}
& \left(\left(1 \otimes W_{G}\right)(a \otimes e \otimes \rho(r))\left(1 \otimes W_{G}^{*}\right) \xi\right)(s, t) \\
& \quad=\left((a \otimes e \otimes \rho(r))\left(1 \otimes W_{G}^{*}\right) \xi\right)(s, t s)=a \delta_{1, s}\left(\left(1 \otimes W_{G}^{*}\right) \xi\right)(s, t s r) \\
& \quad=a \delta_{1, s} \xi\left(s, t s r s^{-1}\right)=(a \otimes e \otimes \rho(r) \xi)(s, t) .
\end{aligned}
$$

Thus for $x \in \mathscr{M}$ we have

$$
\begin{gathered}
\bar{\delta}_{p}(\theta(x))=\operatorname{Ad}\left(1 \otimes W_{G}\right) \cdot(\sigma \otimes \iota)(\delta(x) \otimes e) \\
=(\iota \otimes \sigma)(\delta(x) \otimes e)=(\theta \otimes \iota) \cdot \delta(x)
\end{gathered}
$$

and $\bar{\delta}_{p}$ is conjugate to $\delta$.

Q.E.D. 
Theorem 5.5. Let $\delta_{1}, \delta_{2}$ be coactions of a discrete group $G$ on $\mathscr{M}_{1}$, and $\mathscr{M}_{2}$ respectively, and let $p_{j} \in \mathscr{M}_{j} \otimes \mathscr{Q}\left(L^{2}(G)\right)$ be the projections of Lemma 5. 4. Suppose $\varphi: \mathscr{M}_{1} \bar{\otimes} \mathscr{L}\left(L^{2}(G)\right) \mapsto \mathscr{M}_{2} \bar{\otimes} \mathscr{L}\left(L^{2}(G)\right)$ is an isomorphism with $(\varphi \otimes \iota) \cdot \bar{\delta}_{1}=\bar{\delta}_{2} \cdot \varphi$. Then $\delta_{1}$ and $\delta_{2}$ are conjugate if and only if $\varphi\left(p_{1}\right)=\theta\left(p_{2}\right)$ for some $\theta \in \operatorname{Aut}\left(\mathscr{M}_{2} \bar{\otimes} \mathscr{L}\left(L^{2}(G)\right)\right)$ commuting with $\bar{\delta}_{2}$.

Proof. The necessity is clear.

Conversely, suppose such a $\theta$ exists; if $\sim$ denotes conjugacy, we have $\bar{\delta}_{1} \sim \overline{\boldsymbol{\delta}}_{1, p_{1}} \sim \overline{\boldsymbol{\delta}}_{2, \varphi\left(p_{1}\right)} \sim \overline{\boldsymbol{\delta}}_{2, \theta^{-1} \varphi\left(p_{1}\right)}=\overline{\boldsymbol{\delta}}_{2, p_{2}} \sim \delta_{2}$, using Lemma 5.4 and the fact that $(\theta \otimes \iota) \cdot \bar{\delta}_{2}=\bar{\delta}_{2} \otimes \theta$.

Q. E. D.

Note that by [20], $\mathscr{M} \searrow_{\delta} G$ is injective whenever $\mathscr{M}$ is, so that using Theorems 5.3 and 5.5, the invariants of $\S 1$ become available to distinguish stable conjugacy and conjugacy classes of coactions of discrete amenable groups as soon as the crossed products $\mathscr{M} \rtimes_{\hat{o}} G$ are known to be semifinite. But the discreteness of $G$ yields a simple characterization for the semifiniteness of $\mathscr{M} \rtimes_{\delta} G$ : the semifiniteness of $\mathscr{M} \searrow_{\delta} G$ is equivalent to that of the fixed point algebra $\mathscr{M}^{\delta}$. By duality, except for the obvious restrictions, all characteristic invariants as well as modules can occur as invariants of coaction.

The following examples provide us further constructions of coactions.

Examples 5.6. Let $H, G$ be locally compact second countable topological groups, and let $\varphi: H \mapsto G$ be a continuous homomorphism with kernel $K$, and let $\omega \in Z^{2}(H, \mathbb{T})$. If $\mathscr{R}^{\omega}(H)$ is the von Neumann algebra of the right regular projective $\omega$-representation $\rho^{\omega}$ of $H$, so for that for $\xi \in L^{2}(G)$,

$$
\left(\rho^{\omega}(h) \xi\right)(k)=\omega(k, h) \xi(k h),
$$

then we can obtain a coaction $\delta=\delta(\varphi, \omega)$ of $G$ on $\mathscr{R}^{\omega}(H)$ via

$$
\delta(x)=W_{\varphi}^{*}(x \otimes 1) W_{\varphi}, \quad x \in \mathscr{R}^{\omega}(H),
$$

where $W_{\varphi}$ is the unitary on $L^{2}(H \times G)$ defined by

$$
\left(W_{\varphi} \xi\right)(h, s)=\xi(h, s \varphi(h)) .
$$

Note that $\delta\left(\rho^{\omega}(h)\right)=\rho^{\omega}(h) \otimes \rho(\varphi(h))$, so that the generators of $\mathscr{R}^{\omega}(H)$ $\searrow_{\delta} G$ are given by $\rho^{\omega}(h) \otimes \rho(\varphi(h))$ for $h \in H$ and $1 \otimes M_{f}$ for $f \in L^{\infty}(G)$, 
where $M_{f}$ is the operator of multiplication by $f$ on $L^{2}(G)$. Note that these are precisely the generators of the twisted crossed product $L^{\infty}(G) \rtimes_{\varphi, \omega} H$, where $H$ acts on $G$ through $\varphi$ via right translation, so that by varying $H, G, \varphi$ and $\omega$ one can obtain any desired type for $\mathscr{R}^{\omega}(H) \searrow_{\delta} G$. Also, the dual automorphism group $\hat{\delta}_{r}$ is given by $\hat{\delta}_{r}=$ $\operatorname{Ad}(1 \otimes \lambda(r)), \lambda$ denoting the left regular representation of $G$.

We assume from this point on that $H$ and $G$ are discrete, $H=K \rtimes_{s} G$, and $\omega$ is lifted from a cocycle, also denoted by $\omega$, on $G_{0}$ The unitary $U$ on $L^{2}\left(G ; L^{2}(H)\right)$ given by

$$
(U \xi)(s)=\rho^{\omega}\left(h_{s}\right) \xi(s), \quad \xi \in L^{2}\left(G ; L^{2}(H)\right),
$$

carries $\mathscr{R}^{\omega}(H) \searrow_{\delta} G$ to $\rho^{\omega}(K)^{\prime \prime} \bar{\otimes} \mathscr{L}\left(L^{2}(G)\right)$, and the dual automorphism group to $r \in G \mapsto \mathrm{Ad} \rho^{\omega}\left(h_{r}\right) \otimes \lambda^{\bar{\omega}}(r)$; here $r \in G \vdash h_{r} \in H$ is the inclusion of $G$ in $H$, and $\lambda^{\omega}(r)$ is the projective left regular representation of $G$ i. e.

$$
\left(\lambda^{\bar{\omega}}(r) \xi\right)(s)=\omega\left(r, r^{-1} s\right)^{-1} \xi\left(r^{-1} s\right), \quad \xi \in L^{2}(G) .
$$

Recall that $\lambda^{\bar{\omega}}(r) \lambda^{\bar{\omega}}(s)=\omega(r, s)^{-1} \lambda^{\bar{\omega}}(r s)$; also, since $\omega$ is lifted from $G$, $\rho^{\omega}(K)^{\prime \prime}=\rho(K)^{\prime \prime}$.

If $K$ is abelian, the restriction of the dual automorphism group to the centre of $\mathscr{R}^{\omega}(H) \searrow_{\delta} G$ is realized by the transpose to $\hat{K}$ of the given action of $G$ on $K$, so that for $x \in \hat{K}$, the groups $N_{x}=H_{x}$ of $\S 1$ are given by

$$
N_{x}=\text { the stabilizer in } G \text { of } x \in \hat{K} \text {; }
$$

since the corresponding automorphism of $\mathscr{L}\left(L^{2}(G)\right)$ is implemented by $\lambda^{\bar{\omega}}$, we obtain

$$
\mu_{x}(r, s)=\omega(r, s)^{-1} \text { on } N_{x} \times N_{x}
$$

In this case, since

$$
\lambda^{\bar{\omega}}(r) \lambda^{\bar{\omega}}\left(r^{-1} s r\right) \lambda^{\bar{\omega}}(r)^{*}=\omega\left(r, r^{-1} s r\right)^{-1} \omega(s, r) \lambda^{\bar{\omega}}(s),
$$

the " $\lambda$ " part of the characteristic invariant is given by

$$
\lambda_{x}(r, s)=\omega\left(r, r^{-1} s r\right)^{-1} \omega(s, r) \text { on } N_{x} \times N_{x} .
$$

Thus if $K$ is also finite, coactions $\delta\left(\omega_{1}\right)$ and $\delta\left(\omega_{2}\right)$ arising from different cocycles $\omega_{1}$ and $\omega_{2}$ on $H$ are stably conjugate if and only if $\omega_{1}$ is cohomologous to $\omega_{2}$, since $N_{1}=G$; the case where $K$ is trivial was analyzed in [11] from a somewhat different perspective. However, if $K$ is infinite this no longer need be the case since it may 
happen that all isotropy groups except that at the identity character are trivial.

We turn now to the other extreme, where $K$ is an infinite conjugacy class, or I. C. C. discrete group. In this case $\mathscr{R}(K)$ is a factor; the following result, generalizing [1; Theorem 5.1], guarantees that the dual automorphisms $\hat{\delta}_{r}$ are outer except for the obvious cases i. e. when $r$ itself acts as an inner automorphism of $K$.

Proposition 5.7. Let $K$ be a locally compact separable group such that $\mathscr{R}(K)$ is a factor, let $\alpha$ be an automorphism of $K$, and $\tilde{\alpha}$ the corresponding automorphism of $\mathscr{R}(K)$. Then $\tilde{\alpha}$ is inner if and only if $\alpha$ is inner.

Proof. Note that if $\beta \in \operatorname{Aut}(\mathscr{R}(K))$, then $\beta$ arises from an automorphism of $K$ if and only if $\delta \cdot \beta=(\beta \otimes \beta) \bullet \delta$. So if $\tilde{\alpha}=\operatorname{Ad} u$ with $u \in \mathscr{R}(K)$, we have

$$
\delta \cdot \operatorname{Ad} u=\operatorname{Ad}(u \otimes u) \cdot \delta
$$

and hence

$$
\text { Ad } W_{K}^{*}(u \otimes 1)=\operatorname{Ad}(u \otimes u) W_{K}^{*} \text { on } \mathscr{R}(K) \otimes C .
$$

But then

$$
W_{K}^{*}(u \otimes 1)=t(u \otimes u) W_{K}^{*}
$$

for some

$$
t \in \rho(K)^{\prime} \bar{\otimes} \mathscr{L}\left(L^{2}(K)\right),
$$

and hence

$$
\delta(u)=t(u \otimes u) .
$$

But $\delta(u) \in \mathscr{R}(K) \bar{\otimes} \mathscr{R}(K), \quad$ and $\quad u \otimes u \in \mathscr{R}(K) \bar{\otimes} \mathscr{R}(K)$, so that $t \in$ $[\mathscr{R}(K) \bar{\otimes} \mathscr{R}(K)] \cap\left(\mathscr{R}(K)^{\prime} \bar{\otimes} \mathscr{R}(K)\right)=\boldsymbol{C} \otimes \mathscr{R}(K)$, as $\mathscr{R}(K)$ is a factor; in addition, both $\delta(u)$ and $u \otimes u$ are invariant under the symmetry $\sigma$, so that $t$ is a scalar. But then

$$
\delta(t u)=t u \otimes t u,
$$

so that $t u=\rho(r)$ for some $r \in K$, by [18].

Q.E. D.

We note that even if $\mathscr{R}(K)$ is not a factor, the proof above shows that $\delta(u)=t(u \otimes u)$ with $t \in \mathscr{Z}(K) \otimes \mathscr{Z}(K)$, when $\mathscr{Z}(K)$ denotes the 
centre of $\mathscr{R}(K)$. It also follows that the actions of $H$ on $\mathscr{R}(K)$ determined by arbitrary extensions of the form:

$$
1 \mapsto K \mapsto H \vdash G \mapsto 1
$$

have trivial characteristic invariants whenever $K$ is $\mathbb{I}$. C. C. - this does not occur for general discrete groups $K$ as is seen by easy examples.

Returning to the situation of semidirect products $K \rtimes_{s} G$ and cocycles $\omega \in Z^{2}(G, \mathbb{T})$, we see that the coactions of $G$ on $\mathscr{R}(K)$ determined by actions $s_{1}$ and $s_{2}$, and cocycles $\omega_{1}$ and $\omega_{2}$, are stably conjugate if and only if

$$
N_{1}=N_{2} \text { and } \omega_{1} \mid N_{1} \times N_{1} \text { is cohomologous to } \omega_{2} \mid N_{2} \times N_{2},
$$

where

$$
N_{j}=\left\{\tau \in G: s_{j}(\tau) \in \operatorname{Aut}(K) \text { is inner }\right\}
$$

\section{References}

[1] Behncke, H., Automorphisms of crossed products, Tôhoku Math. J., 21 (1969), 580600.

[2] Connes, A., Outer conjugacy classes of automorphisms of factors, Ann. Éc. Norm. Sup., 8 (1975), 383-419.

[3] Classification of injective factors, cases $\mathrm{II}_{1}, \mathrm{II}_{\infty}, \mathrm{III}_{2}, \lambda \neq 1$, Ann. Math., 104 (1976), 73-115.

[4] Periodic automorphisms of the hyperfinite factor of type $\mathrm{II}_{1}$, Acta Sci. Math., 39 (1977), 39-66.

[5] Connes, A., Feldman, J. and Weiss, B., An amenable equivalence relation is generated by a single transformation, $J$. Ergodic Theory and Dynamical Systems, I (1981), 431-450.

[6] Feldman, J., Hahn, P. and Moore, C. C., Orbit structure and countable sections for actions of continuous groups, Adv. in Math., 28 (1978), 186-230.

[7] Feldman, J. and Moore, C. C., Ergodic equivalence relations, cohomology, and von Neumann algebras, I, Trans. Amer. Math. Soc., 234. (1977), 289-324; II, ibid., 234 (1977), 325-359.

[8] Haagerup, U., The standard form of von Neumann algebras, Math. Scand., 37 (1975), 271-283.

[9] Jones, V., Actions of finite groups on the hyperfinite type $\mathrm{II}_{1}$ factor, Mem. Amer. Math. Soc., 237 (1980).

[10] Jones, V. and Takesaki, M., Actions of compact abelian groups on semifinite injective factors, Acta Math., 153 (1984), 213-258.

[11] Katayama, Y. and Song, G., Ergodic coactions of discrete groups, Math. Japonica, 27 (1982), 159-175.

[12] Mackey, G. W., Point realizations of transformation groups, Illinois J. Math., 6 (1962), 327-335.

[13] Nakagami, Y. and Takesaki, M., Duality for crossed products of von Neumann algebras, Lecture Notes in Math., Springer-Verlag, 731 (1979).

[14] Ocneanu, A., Actions des groupes moyennables sur les algèbres de von Neumann, 
C. R. Acad. Sci. Paris., 291 (1980), 399-401.

[15] Sutherland, C., Cohomology and extensions of von Neumann algebras, I, Publ. RIMS., Kyoto Univ., 16 (1980), 105-133; II, ibid. 135-174.

[16] A Borel parametrization of Polish groups, Publ. RIMS., 21 (1985) 1067-1086.

[17] Takesaki, M., The theory of operator algebras, I, Springer-Verlag, New York-BerlinHeiderberg, 1979.

[18] Duality and von Neumann algebras, Lecture Notes in Math., SpringerVerlag, 247 (1972), 666-779.

[19] Duality for crossed products and the structure of von Neumann algebras of type III, Acta Math., 131 (1973), 249-310.

[20] Wasserman, S., Injective $W^{*}$-algebras, Math. Proc. Cambridge Philos. Soc., 82 (1977), 39-48.

[21] Zimmer, R. J., Amenable ergodic group actions and an application to Poisson boundaries of random walks, J. Functional Analysis, 27 (1978), 350-372.

[22] Hyperfinite factors and amenable ergodic actions, Inventiones Math., 41 (1977), 23-31.

[23] Nakagami, Y., Some remarks on crossed products of von Neumann algebras, Yokohama Math. J., 27 (1979), 141-162. 